\title{
Slingshot mechanism in Orion: Kinematic evidence for ejection of protostars by filaments
}

\author{
Amelia M. Stutz ${ }^{1}$ and Andrew Gould ${ }^{1,2}$ \\ ${ }^{1}$ Max-Planck-Institute for Astronomy, Königstuhl 17, 69117 Heidelberg, Germany \\ e-mail: stutz@mpia.de \\ 2 Dept. of Astronomy, Ohio State University, 140 W. 18th Ave., Columbus, OH 43210, USA \\ e-mail: gould@astronomy.ohio-state.edu
}

Received 16 December 2015 / Accepted 22 March 2016

\begin{abstract}
By comparing three constituents of Orion A (gas, protostars, and pre-main-sequence stars), both morphologically and kinematically, we derive the following conclusions. The gas surface density near the integral-shaped filament (ISF) is very well represented by a power law, $\Sigma(b)=37 M_{\odot} \mathrm{pc}^{-2}(b / \mathrm{pc})^{-5 / 8}$, for the entire range to which we are sensitive, $0.05 \mathrm{pc}<b<8.5 \mathrm{pc}$, of projected separation from the filament ridge. Essentially all Class 0 and Class I protostars lie superposed on the ISF or on identifiable filament ridges farther south, while almost all pre-main-sequence (Class II) stars do not. Combined with the fact that protostars are moving $\lesssim 1 \mathrm{~km} \mathrm{~s}^{-1}$ relative to the filaments, while stars are moving several times faster, this implies that protostellar accretion is terminated by a slingshot-like "ejection" from the filaments. The ISF is the third in a series of identifiable star bursts that are progressively moving south, with separations of several Myr in time and 2-3 pc in space. This, combined with the observed undulations in the filament (both spatial and velocity), suggest that repeated propagation of transverse waves through the filament is progressively digesting the material that formerly connected Orion A and B into stars in discrete episodes. We construct a simple, circularly symmetric gas density profile $\rho(r)=17 M_{\odot} \mathrm{pc}^{-3}(r / \mathrm{pc})^{-13 / 8}$ consistent with the two-dimensional data. The model implies that the observed magnetic fields in this region are subcritical on spatial scales of the observed undulations, suggesting that the transverse waves propagating through the filament are magnetically induced. Because the magnetic fields are supercritical on scales of the filament as a whole (as traced by the power law), the system as a whole is relatively stable and long lived. Protostellar "ejection" (i.e., the slingshot) occurs because the gas accelerates away from the protostars, not the other way around. The model also implies that the ISF is kinematically young, which is consistent with several other lines of evidence. In contrast to the ISF, the southern filament (L1641) has a broken power law, which matches the ISF profile for $2.5 \mathrm{pc}<b<8.5 \mathrm{pc}$, but is shallower closer in. L1641 is kinematically older than the ISF.
\end{abstract}

Key words. ISM: clouds - stars: formation - ISM: structure

\section{Introduction}

Opaque filamentary structures have been recognized in the interstellar medium (ISM) for well over a century. As already pointed out by Barnard, the undulating form and uniform width of these structures cry out for an explanation: "these [structures] are especially striking in $\alpha=19^{\mathrm{h}} 23^{\mathrm{m}}, \delta=+10^{\circ} 25^{\prime}$, where they cover a space over $1^{\circ}$ wide and form a rather complicated system of twistings and turnings of dark lanes... The strange thing about all such lanes is that they always are of uniform width throughout their ramifications. This must have some meaning beyond mere chance" (Barnard 1905). See Fig. 1.

While Barnard himself does not appear to have ever offered such an explanation, it seems difficult to conceive of any other than near-critical magnetic fields, i.e., systems with comparable magnetic and gravitational potential energy. Of course, by now it is well known from cosmological simulations (which are well matched by observations) that gravity alone can produce filamentary structures. However, these never have Barnard's "twistings and turnings" because such morphologies would be unstable under the influence of gravity alone. They require a competition to gravity from a restoring force.

While the general understanding that stars form from collapsing clouds of gas goes back more than $300 \mathrm{yr}$ (Kant 1755), the fact that such clouds are embedded in gas filaments has only

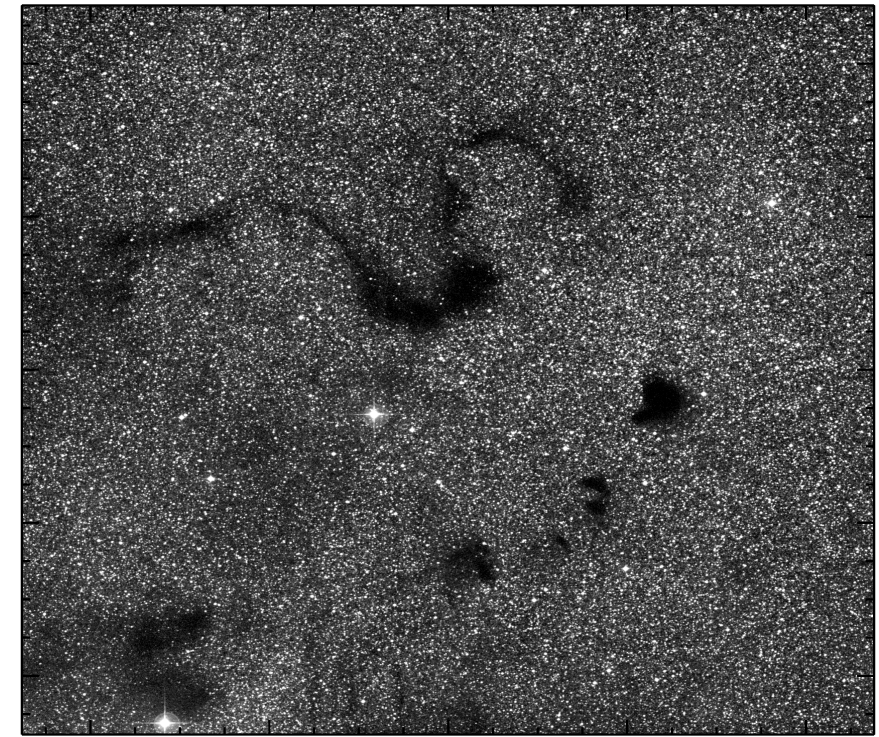

Fig. 1. Photographic image of Barnard 68 from Digitized Sky Survey. Barnard (1905) already argued that the "uniform width" and "twistings and turnings of dark lanes" including this one "must have some meaning beyond mere chance". $\left(57^{\prime} \times 57^{\prime}\right.$, center $\left(17^{\mathrm{h}} 24^{\mathrm{m}},-24^{\circ}\right)$, north up, east left.) 
become clear over the last $50 \mathrm{yr}$ with the discovery, and gradually improving measurement, of Class 0 and Class I protostars directly superposed on filamentary structures in Orion, Aquila, Taurus, etc. (e.g., André et al. 2014; Stutz \& Kainulainen 2015).

The discovery by Heiles (1997) that the Orion A filament (the largest nearby star-forming structure of this kind) is enveloped in a helical magnetic field greatly clarified the nature of these filaments. The observations (their Fig. 24) show magnetic field lines changing direction as they cross the filament, first into then out of the plane of the sky. Since these are one-dimensional (1D) projections of an intrinsically 3D field structure, they cannot be uniquely interpreted by themselves. However, given that circular (or more generally, helical) fields, which would be generated primarily by currents moving along the filaments, are the form that is necessary to confine filaments of approximately uniform thickness, the Heiles (1997) observations constituted a "smoking gun". Moreover, polarization measurements by Matthews \& Wilson (2000) provide information in a second dimension that confirms this picture. That is, under the assumption that this polarization arises from dust grains aligned by either paramagnetic inclusions or radiative torques, the field lines pass over the filament perpendicular to its axis. See their Fig. 1. Subsequent observations confirm these results (Poidevin et al. 2010, 2011). See also Pillai et al. (2015) for dust polarization examples in more distant and massive clouds.

The potential benefits of space-based observations from Spitzer and especially Herschel are two-fold. First, deep observations over a broader range of wavelengths permit studies that go much deeper into protostellar envelopes and hence probe much earlier phases of stellar birth (e.g., Stutz et al. 2010, 2013; Ragan et al. 2012; Pezzuto et al. 2012; Furlan et al. 2014; Safron et al. 2015). Second, the stability of these space-based observations makes it possible to probe to much lower column densities, and thus to understand the gravitational potential that serves as governor to the system in the face of sustained (or repeated episodes of) star formation.

While the first aspect has been systematically exploited, the second is mostly untouched. Ground-based data are intrinsically biased against low-column-density regions in two distinct, if related, ways. First, high and variable atmospheric foregrounds fundamentally limit the precision of surface brightness measurements and so the minimum surface brightness that can be measured. More subtlety, the very process of removing this background (chopping) acts as a high-pass spatial filter and therefore emphasizes the spiny filamentary features at the expense of the much larger low-density structures that govern the overall gravitational potential.

However, because of the high interest of the spiny filaments themselves (e.g., André et al. 2010; Arzoumanian et al. 2011), particularly because these are the immediate sites of star formation, space-based morphological studies have primarily emphasized probing the fine detail of these structures using wavelet analysis or other high-pass filters, which deliberately reproduces the principal characteristic of ground-based data.

Here, we adopt an integrated, empirical approach to this subject. We begin by focusing on the least studied aspect, the overall morphology of the Orion A cloud on the largest scales available from Herschel data. This allows us to estimate the gravitational potential on all scales from the resolution limit (about $0.04 \mathrm{pc}$ ) to $8.5 \mathrm{pc}$. This sets the stage to examine the kinematics of the stars and gas in a new light and to derive far-reaching conclusions.

In particular, we argue for a new slingshot mechanism that "ejects" protostars from the dense filaments that nurtured them, thereby cutting off their accretion of new gas. That is, the filaments are always undergoing transverse acceleration, and the nascent protostars are accelerated with them. When the protostar system becomes sufficiently massive to decouple from the filament, it is released. See Fig. 2 for a schematic diagram of the process. As with a terrestrial hunter's slingshot, no impulse is imparted to the projectile at the moment of release. Rather, it is the filament that accelerates away from the protostar. As with the hunter, so with the Hunter.

The reader with limited time is advised to skip directly to the conclusions.

\section{Power-law morphology of the integral-shaped filament}

As its name implies, the integral-shaped filament (ISF) near the northern end of Orion A (e.g., Bally et al. 1987; Johnstone \& Bally 1999) is characterized by a ridge of gas in the shape of an elongated "S". See Fig. 3. For purposes of this paper, we designate the ISF as $-5.9^{\circ}<\delta<-4.9^{\circ}$ and refer to the remainder of Orion A $\left(-9.1^{\circ}<\delta<-4.9^{\circ}\right)$ as L1641. In order to study the extended morphology of these structures, we first identify a ridgeline of peak gas surface density, using the dust column density map of Stutz \& Kainulainen (2015) as a function of Declination $\delta, \alpha_{\text {ridge }}(\delta)$. We obtain comparable column density maps to those reported by Polychroni et al. (2013) in L1641, Ripple et al. (2013) from CO measurements, and Lombardi et al. (2014) using a combination of near-infrared extinction and Herschel emission. We then define $(x, y)$ positions by

$x=\left(\alpha-\alpha_{\text {ridge }}\right)(\cos \delta) D_{\text {Orion }}, \quad y=\delta D_{\text {Orion }}$,

where $D_{\text {Orion }}=420 \mathrm{pc}$.

We then form cumulative distribution functions of mass for various different widths $w$ as a function of $\delta$

$M(w, \delta)=110 \times 1.34 \sum_{-y_{0}<j<y} \sum_{-w<i<w} M_{i j, \mathrm{dust}}$,

where $y_{0}$ is the southernmost point of the structure in question, $M_{i j \text {,dust }}$ is the mass in dust of the $(i, j)$ pixel, 110 is the assumed hydrogen-to-dust mass ratio, and 1.34 is the assumed ratio of total gas to hydrogen gas. Figure 4 shows this function for several values of $w$. These curves are all well approximated by straight lines (as are the curves for all intervening values of $w$, not shown). This demonstrates that, at a given distance from the ridgeline, the surface density is essentially independent of $\delta$. This permits us to average over all $\delta$ within each structure to find the line density $\lambda(w)$ as a function of enclosing width

$\lambda(w)=\frac{M\left(w, \delta_{+}\right)-M\left(w, \delta_{-}\right)}{D_{\text {Orion }}\left(\delta_{+}-\delta_{-}\right)}$

where $\delta_{ \pm}$are the adopted boundaries of the structures, indicated above. For the ISF, this cumulative distribution function is very well approximated by a straight line (on a log-log plot, Fig. 5), namely,

$\lambda(w)=K\left(\frac{w}{\mathrm{pc}}\right)^{\gamma} ; \quad K=385 \frac{M_{\odot}}{\mathrm{pc}}, \quad \gamma=\frac{3}{8}$.

We note that this power-law behavior extends to $w= \pm 8.5 \mathrm{pc}$, i.e., $\pm 1.2^{\circ}$, which is the approximate limit of the underlying Stutz \& Kainulainen (2015) map. We note that the L1641 cumulative distribution is a broken power law. For $w>2.5 \mathrm{pc}$, both the slope and normalization are consistent with those of the ISF (Eq. (4)), while at closer separations the projected density falloff is significantly more shallow $\gamma(\mathrm{L} 1641,<2.5 \mathrm{pc})-1=-1 / 2$ versus $-5 / 8$ for the ISF. 
A. M. Stutz and A. Gould: Slingshot mechanism in Orion: Kinematic evidence for ejection of protostars by filaments

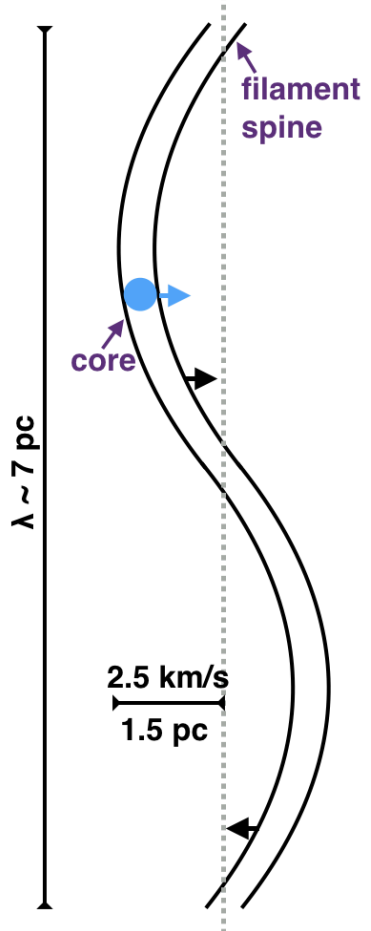

$\mathbf{t}=\mathbf{0}$

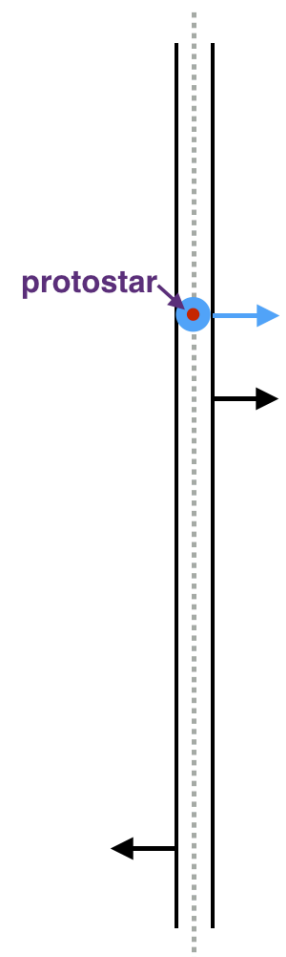

$t=1$

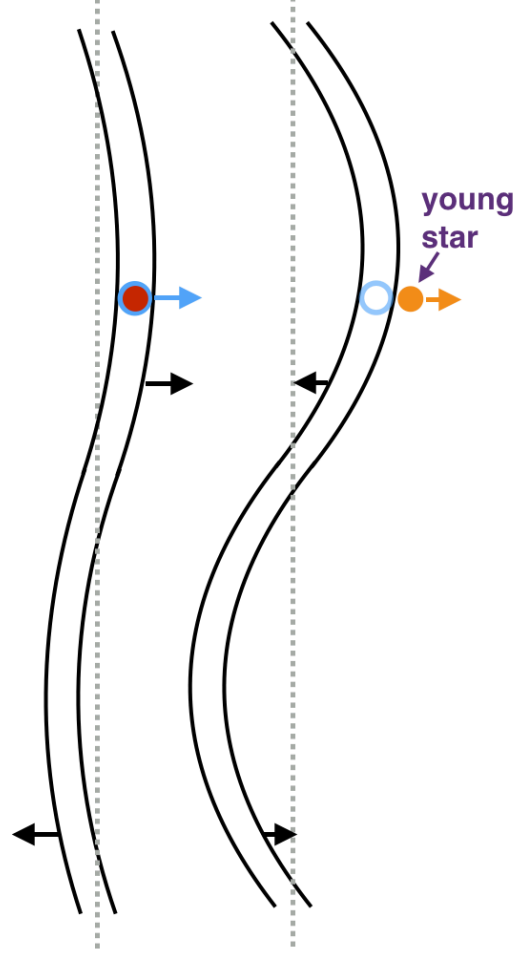

$\mathbf{t}=\mathbf{2}$

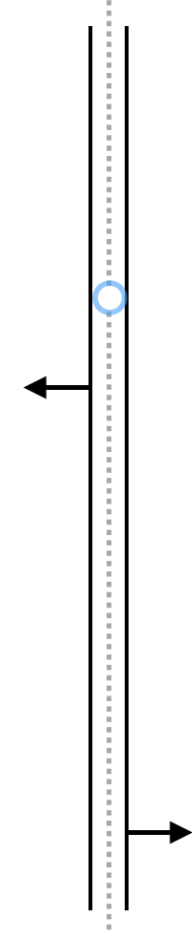

$\mathbf{t}=3$

Fig. 2. Cartoon showing time series of slingshot "ejection" of a protostar from the ISF. Left to right: (1) core forms in filament; (2) core evolves into protostar and remains mechanically entrained in accelerating filament; (3) protostar continues to gain mass but remains in filament due to relatively low instantaneous filament acceleration; (4) filament reaches maximum acceleration (toward left) so that inertia of protostar causes it to leave filament, decoupling from dense gas cradle; (5) "ejected" young star moves away from filament.

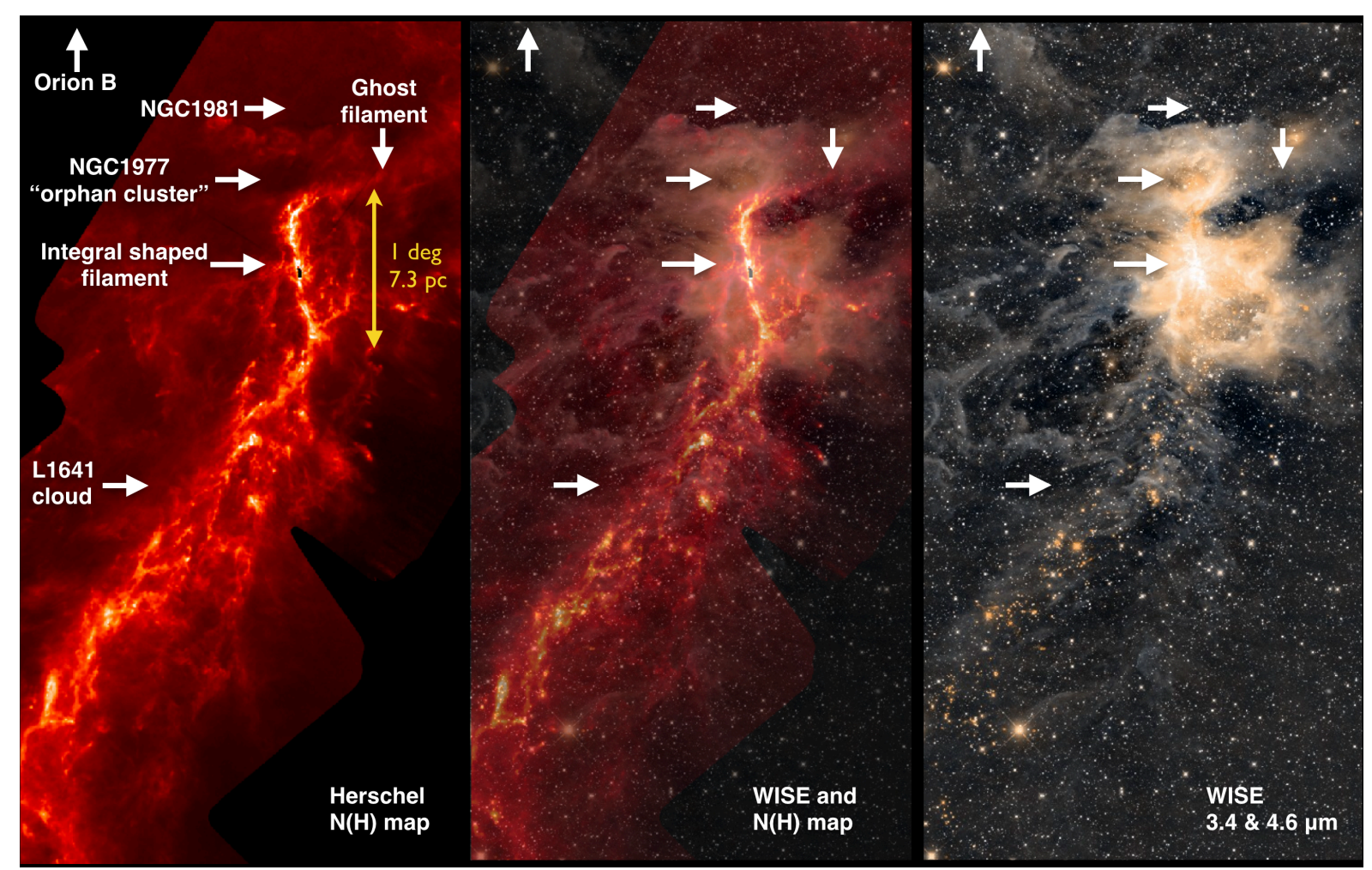

Fig. 3. Location of the regions and structures discussed throughout this paper shown over the Herschel column density map (left), the WISE combined image (Lang 2014, right) and the combination of the two (middle). 

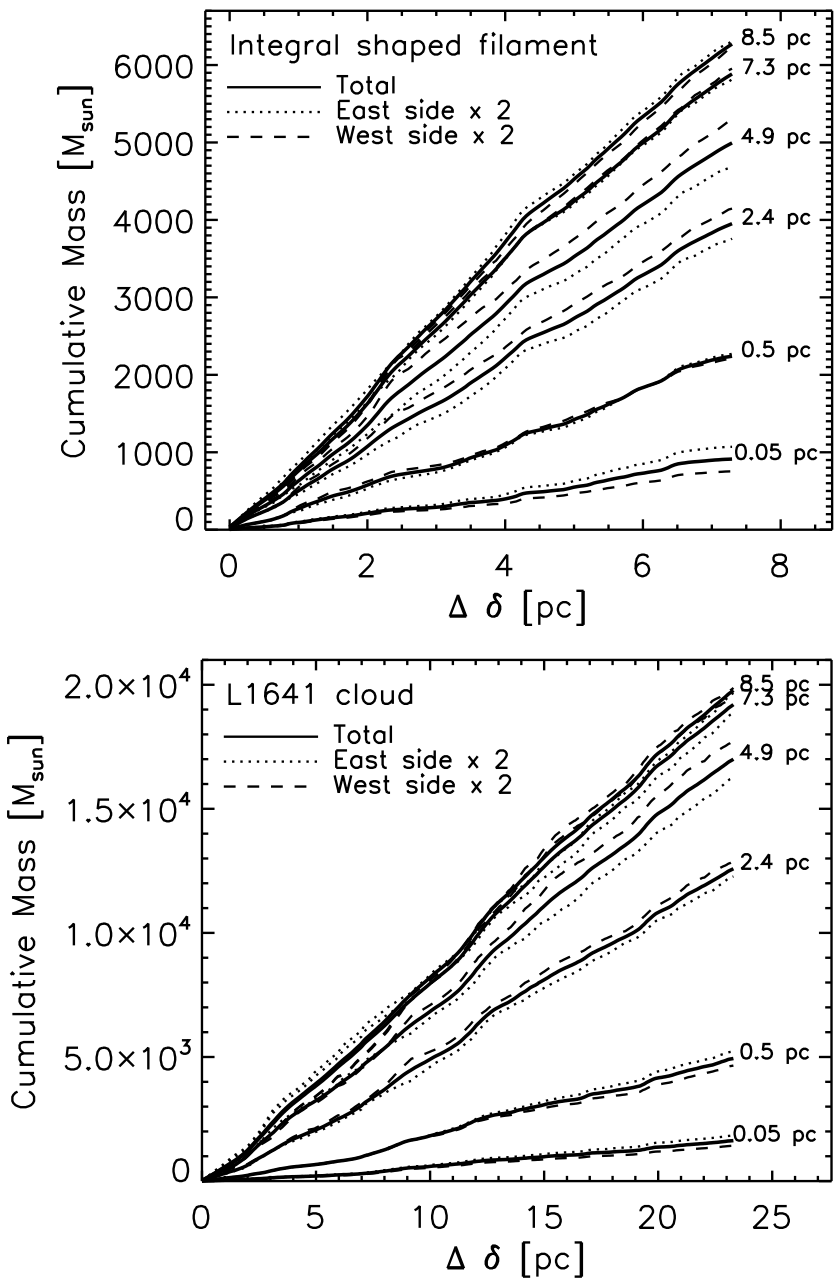

Fig. 4. Cumulative distributions of mass within various projected separations $w$ from the density ridgeline. Top and bottom panels show the integral-shaped filament (ISF) and remaining region of Orion A (L1641) respectively. The cumulative distributions start at the southern boundary of each structure. The separate cumulative distributions for the east $(-w<x<0)$ are west $(0<x<+w)$ are shown in different line types. For ease of comparison (and clarity) these are multiplied by two.

\section{Inferred volume density and gravitational potential}

Inspired by the simplicity of Eq. (4), we assume axial symmetry about the ridgeline. Then, after some algebra, we find local volume densities of ${ }^{1}$

$\rho(r)=\frac{\gamma(-\gamma / 2) !}{2(-\gamma / 2-1 / 2) !(-1 / 2) !} \frac{K}{\mathrm{pc}^{2}}\left(\frac{r}{\mathrm{pc}}\right)^{\gamma-2}$,

and consequently enclosed line density,

$$
\begin{aligned}
& \Lambda(r)=\int_{0}^{r} 2 \pi r^{\prime} \mathrm{d} r^{\prime} \rho\left(r^{\prime}\right)=f(\gamma) \lambda(r) \\
& f(\gamma) \equiv \frac{(-\gamma / 2) !(-1 / 2) !}{(-\gamma / 2-1 / 2) !} \rightarrow 0.711,
\end{aligned}
$$

where the arrow indicates evaluation according to the measured parameters. For locations close to the filament, the acceleration

1 Almost all integrals in this paper are reducible to $\int_{0}^{1} \mathrm{~d} x x^{a}(1-x)^{b}=$ $a ! b ! /(a+b+1) !$

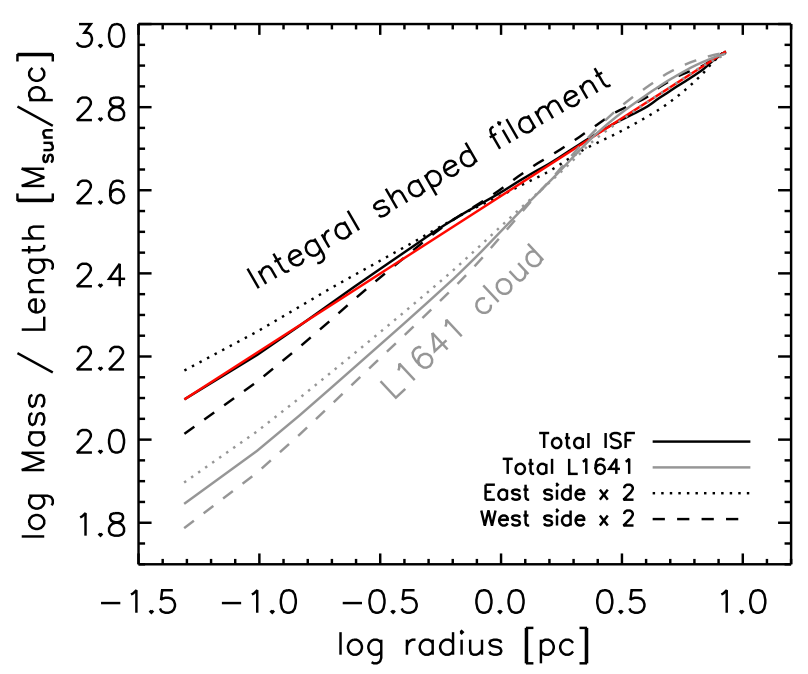

Fig. 5. Enclosed projected mass per unit length as a function of enclosing width $w$, for the ISF and L1641, shown separately. As in Fig. 4, the east and west subsets are shown separately (after multiplying by 2). The ISF is extremely well approximated by a pure power law, which is explicitly indicated as a red line. L1641 shows the same power law (both slope and normalization) for $2.5 \mathrm{pc} \lesssim w<8.5 \mathrm{pc}$, but the surface density falls off substantially more slowly in the inner $2.5 \mathrm{pc}$.

is (by Gauss's Law) $a=2 G \Lambda / r$, and hence the gravitational potential is

$$
\begin{aligned}
& \Phi(r)=\int_{0}^{r} \mathrm{~d} r^{\prime} a\left(r^{\prime}\right)=\eta(\gamma) G \lambda(r) \rightarrow 6.3\left(\mathrm{~km} \mathrm{~s}^{-1}\right)^{2}\left(\frac{r}{\mathrm{pc}}\right)^{\gamma} \\
& \eta(\gamma) \equiv \frac{2(-\gamma / 2) !(-1 / 2) !}{\gamma(-\gamma / 2-1 / 2) !} \rightarrow 3.79 .
\end{aligned}
$$

We note that while the local density and the gravitational acceleration diverge as $r \rightarrow 0$, the potential is well behaved. This means in particular that while the density profile must flatten at some point, kinematics outside of the innermost pixel that is measured (at about $10000 \mathrm{AU}$ ) are insensitive to these details.

Next we calculate the time required for a star, initially at rest at radius $r_{\max }$ to fall to zero,

$\Delta t=\int_{0}^{r_{\max }} \mathrm{d} t(r)=g(\gamma) \frac{r_{\max }}{\sqrt{2 \Phi\left(r_{\max }\right)}}$

where

$g(\gamma)=\frac{(1 / \gamma) !(-1 / 2) !}{(1 / \gamma-1 / 2) !} \rightarrow 3.03$

and

$\mathrm{d} t=\frac{\mathrm{d} r}{v(r)}$,

where

$v(r)=\sqrt{2\left(\Phi\left(r_{\max }\right)-\Phi(r)\right)}$,

is the speed of a particle falling from $r_{\max }$ to $r$. Then, the mean value of any function $F(r)$ over the orbit is

$\langle F\rangle=\frac{\int_{0}^{r_{\max }} \mathrm{d} t(r) F(r)}{\Delta t}$.

In particular, the mean speed is

$\langle v\rangle=\frac{r_{\mathrm{max}}}{\Delta t}=\frac{\sqrt{2 \Phi\left(r_{\mathrm{max}}\right)}}{g(\lambda)} \rightarrow 1.2 \mathrm{~km} \mathrm{~s}^{-1}\left(\frac{r_{\mathrm{max}}}{\mathrm{pc}}\right)^{3 / 16}$, 


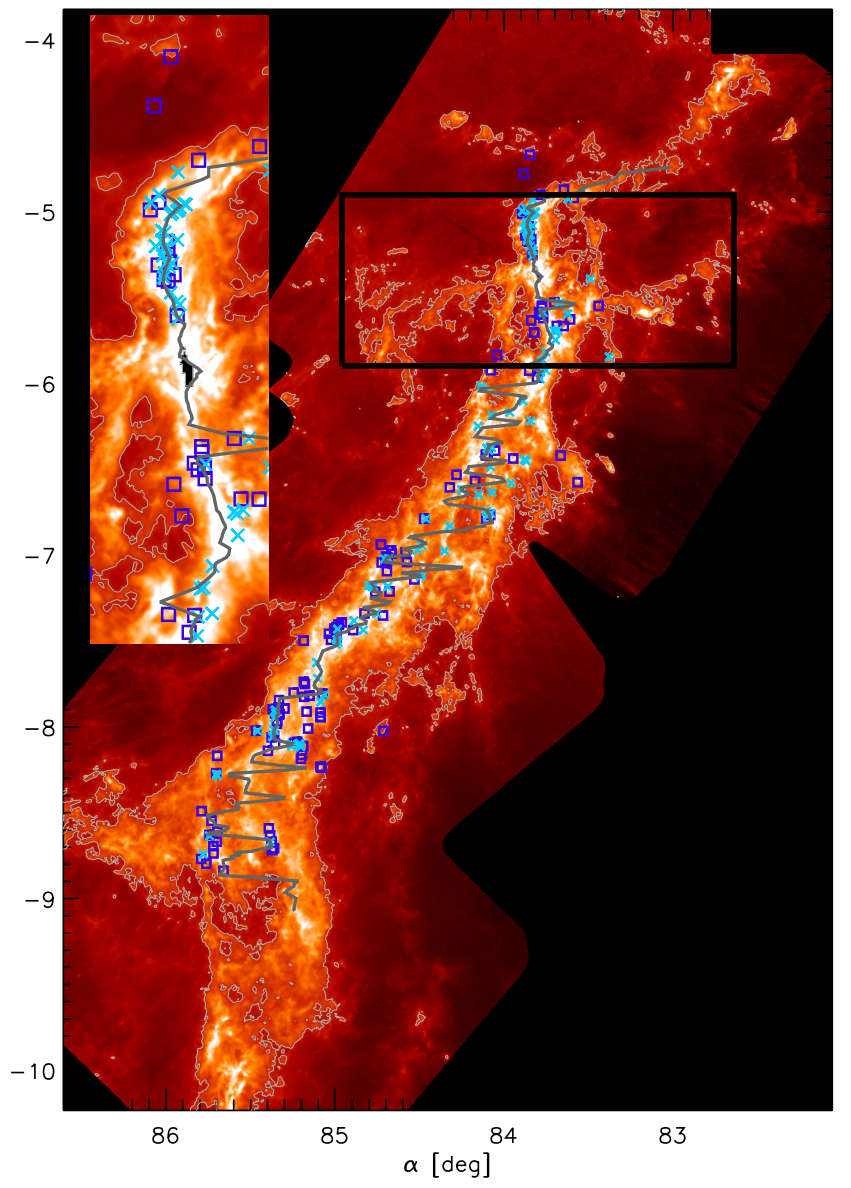

Fig. 6. Positions of protostars (Furlan et al. 2016) compared to gas density as traced by dust emission (Stutz \& Kainulainen 2015). Protostars lie almost exclusively on the ridges of columns. In the north, they are confined to the integral-shaped filament (ISF), which is the dominant structure. In the south, the filamentary structure is more complex. Ordinate is $\delta$ [deg]. Protostars with (without) radial velocity measurements are shown in cyan (blue). The approximate region used to calculate the potential is indicated with a black box of size $\sim 19 \times 7.3 \mathrm{pc}^{2}$.

while the mean radius is

$\langle r\rangle=\frac{r_{\max }}{2} \frac{(2 / \gamma) !}{(2 / \gamma-1 / 2) !} \frac{(1 / \gamma-1 / 2) !}{(1 / \gamma) !} \rightarrow \frac{r_{\max }}{\sqrt{2}}$

where the last step is strictly valid only in the limit of $\gamma \ll 1$, but in practice is accurate to $2 \%$ at $\gamma=3 / 8$. For completeness, we note that the $n$th velocity moment is given by

$\frac{\left\langle v^{n}\right\rangle}{\left[\Phi\left(r_{\max }\right)\right]^{n / 2}}=2^{n / 2} \frac{(n / 2-1 / 2) !(1 / \gamma-1 / 2) !}{(-1 / 2) !(1 / \gamma+n / 2-1 / 2) !}$,

so that in particular, for $n=2$, the right-hand side is $2 \gamma /(2+\gamma) \rightarrow$ 0.32 .

\section{Orion A: Morphology and kinematics}

\subsection{Morphology: Gas vs. protostars vs. stars}

Figures 6 and 7 show the gas column density as traced by dust emission in Orion A (Stutz \& Kainulainen 2015) with, respectively, Class 0 and Class I protostars (Furlan et al. 2016) and pre-main-sequence Class II stars (Megeath et al. 2012) superposed. The pre-main-sequence stars (hereafter stars) shown

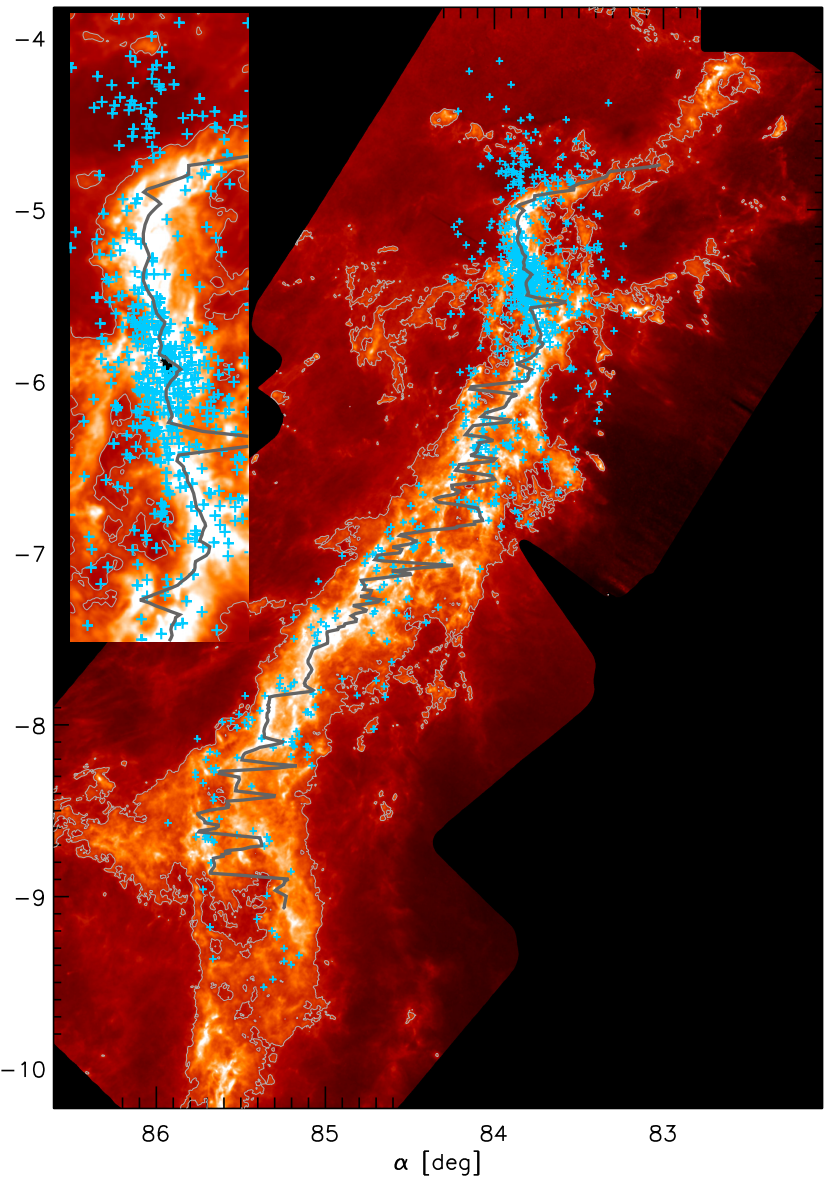

Fig. 7. Positions of pre-main-sequence Class II stars (hereafter stars; Megeath et al. 2012) compared to gas density as traced by dust emission (Stutz \& Kainulainen 2015). Only stars with radial velocities from Apogee are shown. In contrast to the protostars (Fig. 6) the stars have essentially all left the filament (but see Fig. 11). This dichotomy suggests that protostar accretion of ambient gas is terminated by ejection from the gas columns.

are restricted to the subsample with Apogee (Majewski et al. 2015) radial velocities for ease of comparison with the figures in Sect. 4.2. All the protostars are shown, but those without radial velocities are displayed in a darker color. The ridgeline is formed by finding the $\alpha$ of the pixel with peak emission at each $\delta$ in the $4 \times 4$ pixel (final pixel scale of $24^{\prime \prime}$ ) rebinned dust map.

The key morphological features are the following:

1 The protostars almost all sit on narrow gas-column ridges, while the stars are either more broadly distributed near the ridges, or are displaced from the ridges altogether.

2 The gas, protostars, and stars all have highest density along integral-shaped filament (ISF, $\delta \sim-5.5^{\circ}$ ).

3 There is a strong knot of stars, NGC 1977, just north of ISF, which is almost devoid of gas, and which we therefore dub the orphan cluster.

4 There is a tenuous gas ghost filament extending west and then northwest from northern tip of the ISF.

From the fact that the great majority of protostars, which are by definition deeply embedded in dense gas, are superposed on narrow gas column ridges, we can conclude that their envelopes are lost either before or almost immediately after they lose access to the dense gas column. This directly raises the question of whether there is a substantial population of stars within the gas 


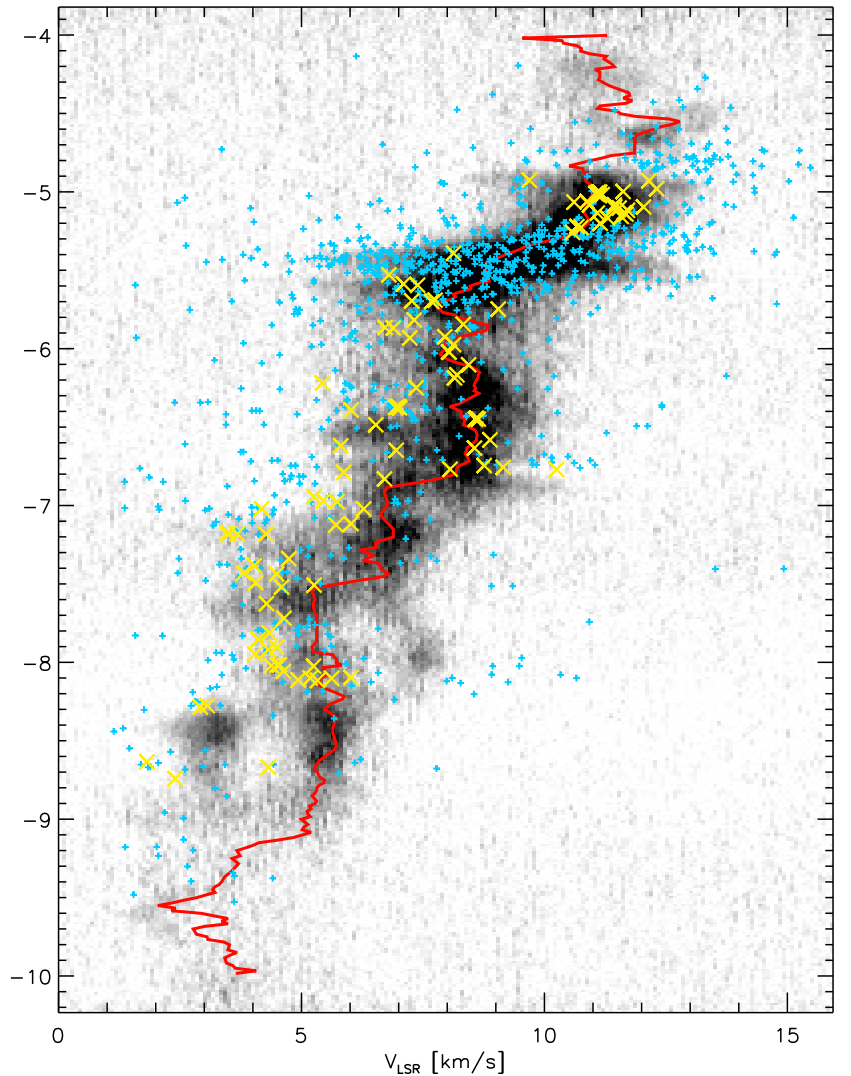

Fig. 8. Position-velocity diagram of ${ }^{13} \mathrm{CO}$ gas (grey-scale), protostars (yellow $\times$-symbols), and stars (blue + -symbols). The red line indicates the ${ }^{13} \mathrm{CO}$ gas ridgeline (see 4.2 ). Gas velocity profile is integrated over $\alpha$. Protostars generally have velocities very close to the peak velocity of the local gas, whereas stars are further displaced. That is, stars have both greater kinetic and greater potential energy than the protostars relative to their local gas columns. Ordinate is $\delta$ [deg].

column ridges. If protostellar envelopes are lost before leaving the filament then there should be such a population, while if they leave immediately after then there should not.

Although it is not proved from Fig. 7 alone, there are in fact essentially no stars in Fig. 7 that sit directly on the gas column ridges. That is, particularly for the ISF, there are a considerable number of stars close to the filament, so that just by chance some will be projected directly on the narrow filament spine (i.e., the location of most of the protostars). However, we will show below, using radial velocity data, that these projections are indeed almost all by chance. These two facts (one demonstrated and one anticipated) together argue that the Class I (embedded) phase is ended by removal of the protostar from the gas column ridge, rather than by somehow exhausting or cutting off supply from the local gas column.

Since these stars used to be protostars, they were formerly lying directly on the gas-column ridge, just as the present-day protostars are. This would be inconsistent with Newton's First Law unless either the stars or the gas column (or both) have accelerated between their Class I phase and today. If the gas were not accelerating, there would be no way to accelerate the stars, since the line-density of protostars and space density of stars are at least an order of magnitude too low to induce transverse motions of the required amplitude, particularly in the southern filament. Hence, we conclude that the filament is undergoing accelerated motion transverse to the filament. We will estimate the amplitude of this motion further below.
The orphan cluster (NGC 1977; Peterson \& Megeath 2008) lying just north of the ISF must have formed out of filamentary gas, just like all the other stars. In principle, these could have been ejected (accelerated) from the location of the present-day ISF. If this is the case, however, it will soon become apparent from Gaia data, since the implied proper motions are of order $1 \mathrm{mas} \mathrm{r}^{-1}$. More likely, the ISF extended to the current midpoint of the orphan cluster a few Myr ago when these stars formed and has since been dispersed or moved. The extended ghost filament to the west is a good candidate for the fate of this putative extension of the ISF. In this case the torques propagating northward through the filament encountered suddenly weakening restoring forces as they exited the ISF and simply ripped the filament away from its former trajectory toward Orion B.

While the orphan cluster is heavily populated by disk sources (i.e., those found by Megeath et al. 2012 to have Spitzer infrared (IR) excesses) there is another cluster (NGC 1981), which lies 3 pc further to the north (hence closer to Orion B), and which was identified by Pillitteri et al. (2013) as containing relatively few Megeath et al. (2012) disk sources but a much larger number of photospheric (Class III) stars, meaning that it is older.

Hence, the overall picture is of repeated episodes of starcluster formation, each triggered by a transverse wave that propagates northward through the filament. Each episode ends in a violent contraction of the filament at its northern end (similar to the ISF today), which both ignites an episode of star formation and then disperses the loose end of the filament that formerly connected Orion A and Orion B.

\subsection{Kinematics: gas vs. protostars vs. stars}

Figure 8 is a position-velocity diagram for the stars and gas shown in Fig. 7 and for the subset of the protostars shown in Fig. 6 for which radial velocity (RV) data are available. The stellar RVs are taken from the Apogee catalog (Majewski et al. 2015; Da Rio et al. 2016). See also Hacar et al. (2016). As mentioned in Sect. 4.1 these are restricted to stars having disks as classified by Megeath et al. (2012).

The initial sample has 1030 stars. Roughly $1 / 4$ of these have multiple measurements and of these 15 show scatter in excess of $2 \mathrm{~km} \mathrm{~s}^{-1}$ and are removed as binaries. Because the RV distribution of this sample is extremely compact, the 49 (out of the remaining 1015) stars with $\mathrm{RV}$ s larger than $7 \mathrm{~km} \mathrm{~s}^{-1}$ from the median are removed from the sample (as being probable background contaminants, but perhaps binaries). We note that the Apogee sample is restricted to $H<12.5$, which may create a subtle but important bias to which we return below.

The protostar RVs come from an online catalog (Di Francesco et al., in prep.) of HOPS sources, which contain independent measurements derived from $\mathrm{NH}_{3}$ and $\mathrm{HC}_{5} \mathrm{~N}$. If these agree to within $0.5 \mathrm{~km} \mathrm{~s}^{-1}$ then the $\mathrm{NH}_{3}$ measurement is accepted. Only one star is eliminated by this check. Of the 248 protostars in Fig. 6, 111 have RV measurements. The $\mathrm{NH}_{3}$ velocities agree well with independent $\mathrm{N}_{2} \mathrm{H}^{+}$measurements (Tatematsu et al. 2008, 2016) in the ISF, see Fig. 9.

The gas velocities are derived from ${ }^{13} \mathrm{CO}(2-1)$ (Nishimura et al. 2015) by collapsing the 3D data cube in ( $\alpha, \delta, v_{r}$ ) along the $\alpha$ axis. The ridgeline is formed by finding the velocity of the pixel with peak emission at each $\delta$ and then boxcar smoothing with a length of 4 pixels corresponding to $4^{\prime}$. The ${ }^{13} \mathrm{CO}$ velocities and ridgeline morphology are compared to the $\mathrm{N}_{2} \mathrm{H}^{+}$measurements in Fig. 9. In the right panel we show the $\mathrm{CO}$ gas ridgeline compared to the $\mathrm{N}_{2} \mathrm{H}^{+}$velocity centroid calculated within $0.5 \mathrm{~km} \mathrm{~s}^{-1}$ the isolated hyperfine component 

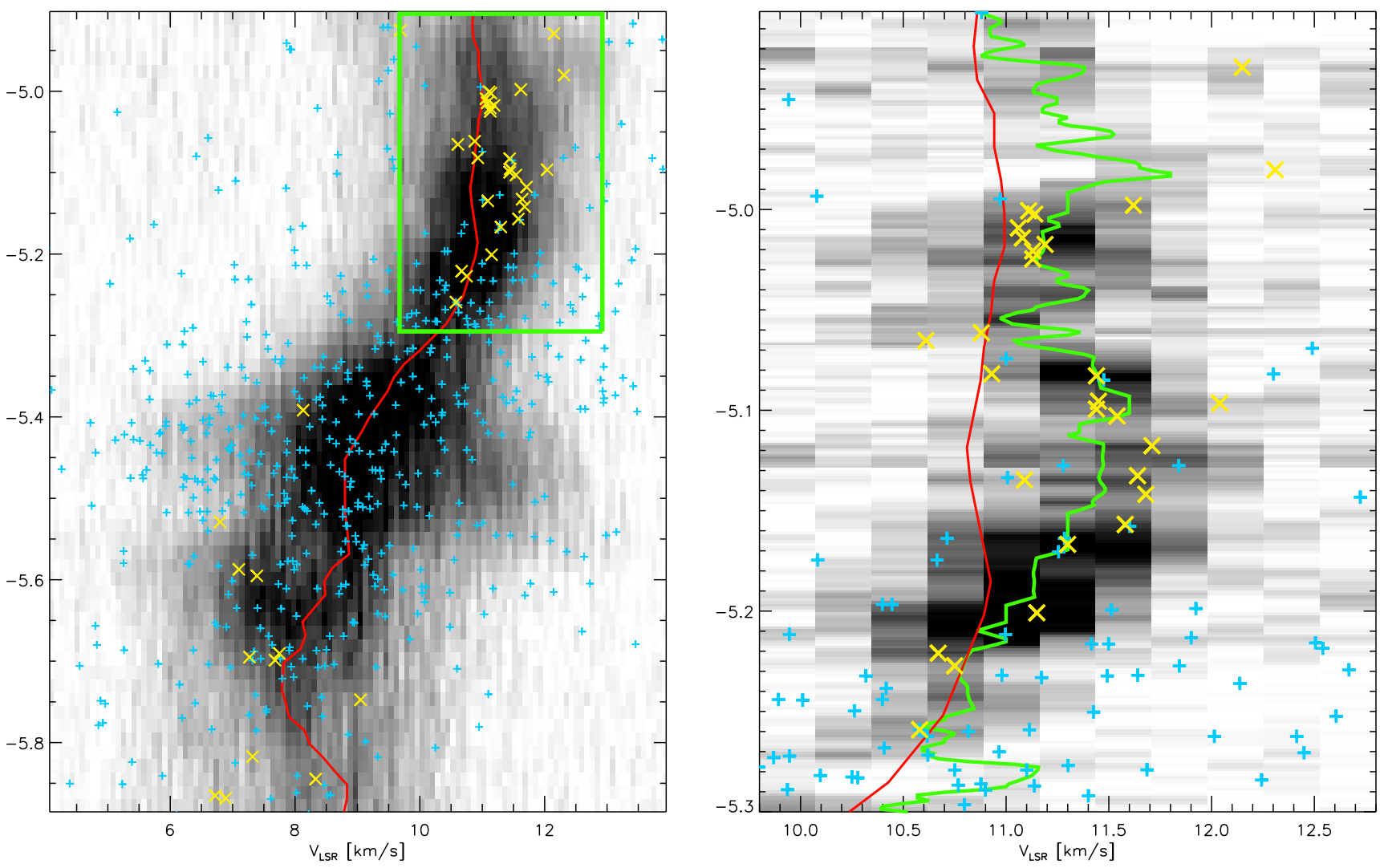

Fig. 9. Left: zoom-in of position-velocity diagram shown in Fig. 8. Green box indicates the area in the diagram on the right. Right: $\mathrm{N}_{2} \mathrm{H}^{+}$positionvelocity diagram of the northern portion of the ISF. Red line is the ${ }^{13} \mathrm{CO}$ gas ridgeline while the green line shows the velocity centroid of the $\mathrm{N}_{2} \mathrm{H}^{+}$ line. The protostars (yellow $\times$-symbols) have velocities that are slightly displaced from the $\mathrm{CO}$ gas but directly associated with $\mathrm{N}_{2} \mathrm{H}^{+}$. Ordinate is $\delta[\operatorname{deg}]$.

line peak. The protostars are substantially closer to the $\mathrm{N}_{2} \mathrm{H}^{+}$ velocity centroid than the $\mathrm{CO}$ gas ridgeline. The $\mathrm{N}_{2} \mathrm{H}^{+}$map coverage and sensitivity prevents detailed analysis of larger areas of the ISF or L1641.

The main features of Figs. 8 and 9 are the following:

1 The protostars are displaced from the gas column in RV by $\lesssim 1 \mathrm{~km} \mathrm{~s}^{-1}$.

2 The stars are also generally displaced from the gas column but by several $\mathrm{km} \mathrm{s}^{-1}$.

3 The gas column exhibits undulations in RV, particularly near the ISF, but also near $\delta \sim-6.5^{\circ}$, i.e., qualitatively similar to the morphological undulations.

4 The orphan cluster (north of the ISF) is dispersed similarly to other stars.

While it is clear that the stars are overall more displaced from the filament than the protostars in both position and velocity, it is not yet proved that there is no sub-population of stars that mimic the protostars in being close to the filament in both these phase-space coordinates. To begin addressing this question we show in Fig. 10 the offsets in velocity versus offsets in position for three populations, protostars in the ISF, stars in the ISF, and stars in L1641. We reserve discussion of most of the features of these figures for Sect. 5. Here we just point out that the ISF stars form a broad 2D distribution in this diagram, especially compared to the ISF protostars, similar to the distributions observed by Foster et al. (2015) in the NGC 1333 region of Perseus. This confirms that there is no significant subpopulation among the sample of Megeath et al. (2012) IR-excess (Class II) stars with
Apogee (Majewski et al. 2015) radial velocities that "hugs" the filaments in the manner displayed by the protostars.

The fact that the protostars have substantially lower specific kinetic energy (in addition to having lower potential energy, as indicated in Sect. 4.1) confirms that nascent stars receive a substantial kick between their protostellar and stellar phases. As discussed above, a natural explanation for this tight correlation between evolutionary phase and total specific energy is that these kicks remove the protostars from the gas rich column in which they were born, thereby shutting off accretion. However, we also consider the alternative explanation that gas accretion shuts down by some self-generated process and the protostar-turnedstar leaves the column by some as yet unspecified process at a later time. This alternative appears to be strongly contradicted by the absence of stars superposed on the ridges of gas columns.

However, this seeming evidence could in principle be partly the result of selection. Class II stars that remained embedded in dusty columns would be fainter in $H$-band due to extinction and so much less likely to be observed by Apogee, given its $H<12.5$ limit. In Fig. 11, we therefore show the Megeath et al. (2012) stars that were not observed by Apogee. For the most part, these excluded stars are not lying on the spines of filaments, which reflects the fact that there are heterogeneous reasons why Megeath et al. (2012) stars were not observed by Apogee. However, there may be some preference for stars very close to filaments, relative to Fig. 7.

We therefore further investigate this possible selection bias in Fig. 12, where we compare the $H$-band magnitude distribution of all Megeath et al. (2012) IR-excess (Class II) stars that lack Apogee RVs, with two subsets that lie projected close to 

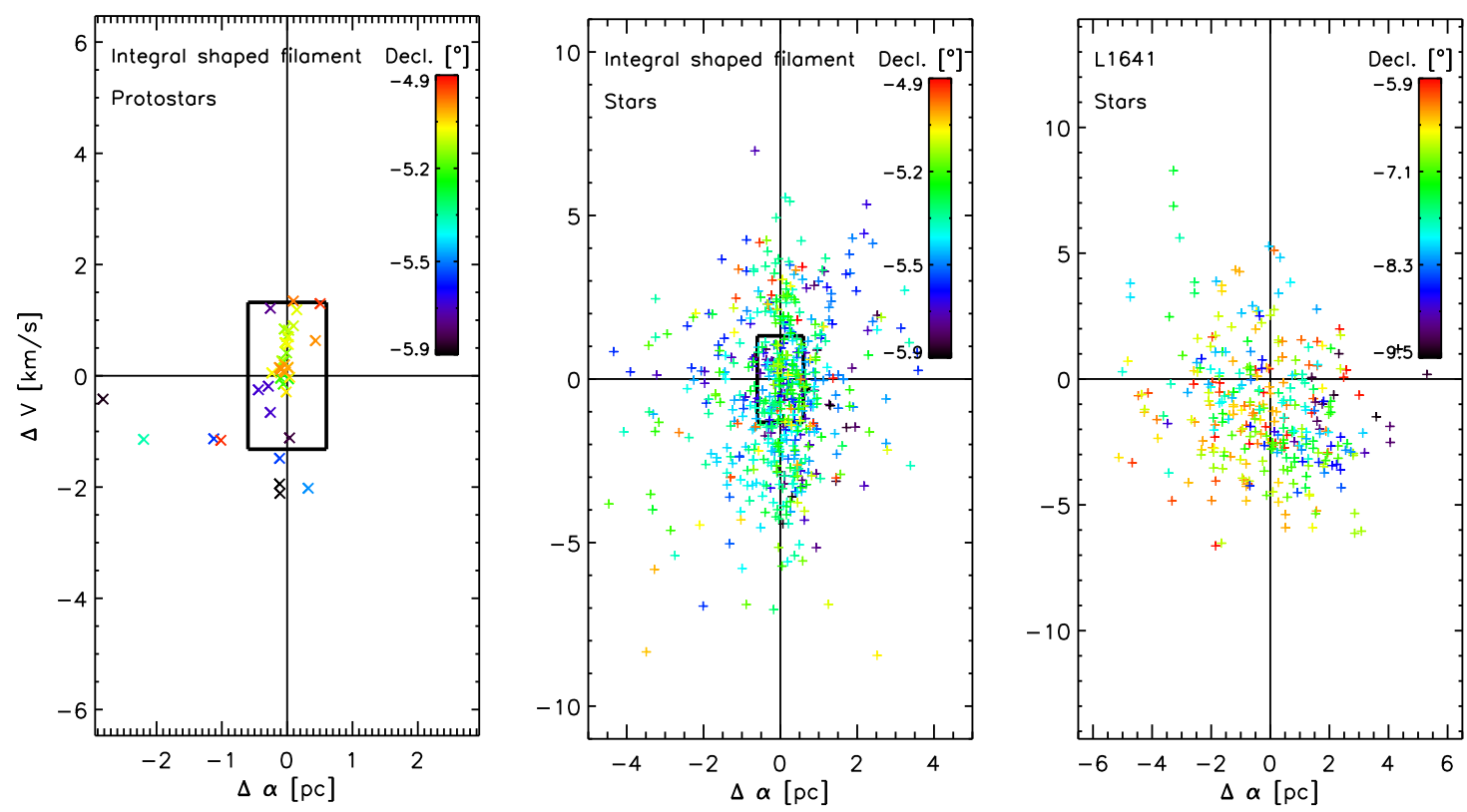

Fig. 10. Offsets of ISF protostars (left), ISF stars (center), and L1641 stars (right) from the gas ridgelines in $\alpha$ (Figs. 6 and 7) and RV (Fig. 8). The protostars are shown only for the ISF because of the difficulty of capturing the filament structure further south by simple prescriptions. Points are color-coded north-to-south $\leftrightarrow$ red-to-blue. Ratio of axis scales is 1 Myr. Hence, the vertically-elongated structure of these diagrams indicates timescales $\ll 1 \mathrm{Myr},<1 \mathrm{Myr}$, and $\sim 1 \mathrm{Myr}$ for the three diagrams, respectively. These could be either lifetime or turnaround time. The stellar distributions (both ISF and L1641) are broadly distributed, with no obvious filament-hugging subpopulations, like the ISF protostars. However, they also show significant lumpiness demonstrating incomplete relaxation. The protostars show some severe clumping, with a high density near the origin, in particular.

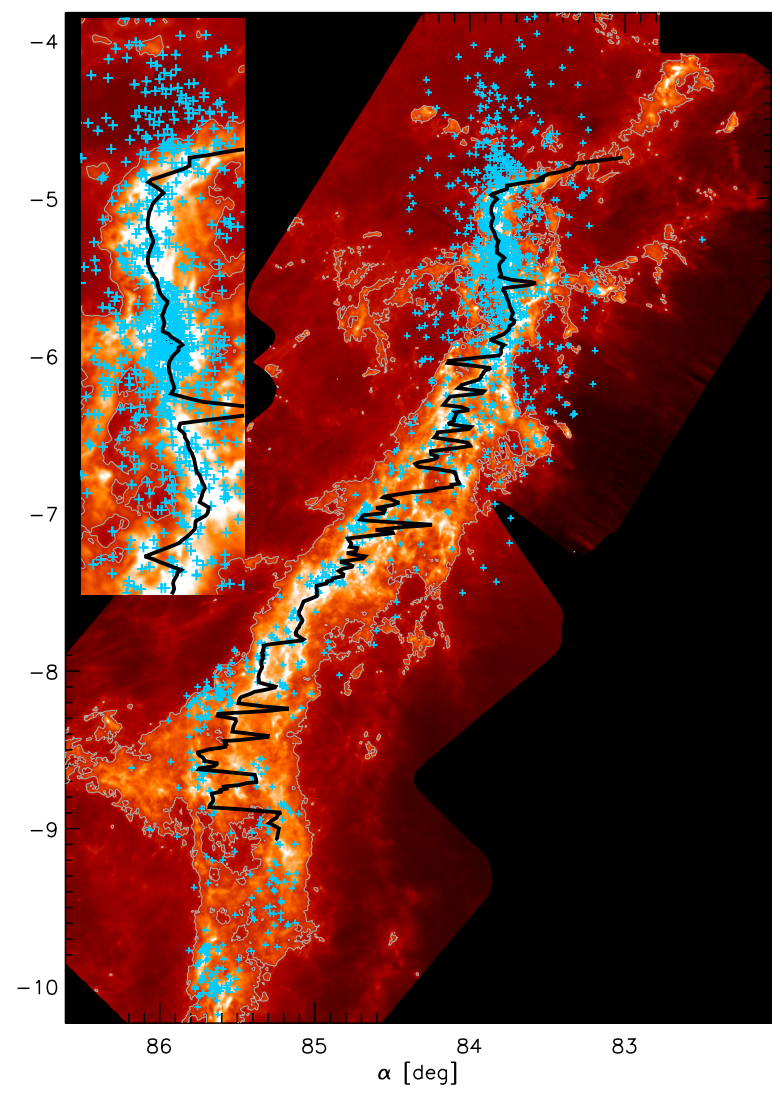

Fig. 11. Star positions relative to gas for stars without Apogee RVs, i.e., the stars that are excluded from Fig. 7. We study these non-Apogee stars to determine whether the Apogee (Majewski et al. 2015; Da Rio et al. 2016) sample is systematically biased against stars lying within the dense spiny columns due to increased extinction. See Fig. 12. Ordinate is $\delta$ [deg]. the filament ridgeline, within, respectively 0.2 and $0.05 \mathrm{pc}$. The $0.2 \mathrm{pc}$ subsample looks identical to the (scaled) full sample, except that it is noisier. Hence, if there is any filament-extinctioninduced bias in the Apogee sample, it must be weak. Nevertheless, given the importance of the conclusions that we derive from the near (or complete) absence of stars within the filamentary spines, we believe that it would be valuable to obtain RVs at least for the $0.05 \mathrm{pc}$ sample. Reliable Apogee spectra can be obtained at substantially lower signal-to-noise than the nominal threshold (Ness et al. 2015), and this applies even more so if the main goal is to obtain RVs.

\section{Characteristic speeds and times in Orion A}

Figure 10 shows RV vs. $\alpha$ offsets from the velocity and position ridgelines for three populations ISF protostars, ISF stars, and L1641 stars. It was introduced in Sect. 4.2 in order to help assess whether stars lying projected close to filamentary spines are actually in them. Here we consider other implications of this figure.

The first point is that the shape of these distributions are, respectively, highly elongated in the RV directions (ISF protostars), moderately elongated (ISF stars), and slightly elongated (L1641 stars). The axis scales are in ratios of $\mathrm{pc} /\left(\mathrm{km} \mathrm{s}^{-1}\right) \simeq$ $1 \mathrm{Myr}$ in all three cases. This implies characteristic timescales of, respectively, «1 Myr, <1 Myr, and $\lesssim 1$ Myr. These timescales indicate either ages or turnaround times.

One way to distinguish between these possibilities is to examine the phase-space distribution in these diagrams, which are color-coded by $\delta$ in order to evaluate clumpiness in all three phase-space coordinates. If the stars or protostars had typically orbited once or more (thereby impressing a turnaround timescale on the diagrams) then they should be well mixed in the RV vs. $\Delta \alpha$ plots along lines passing through the origin. This is clearly 
A. M. Stutz and A. Gould: Slingshot mechanism in Orion: Kinematic evidence for ejection of protostars by filaments
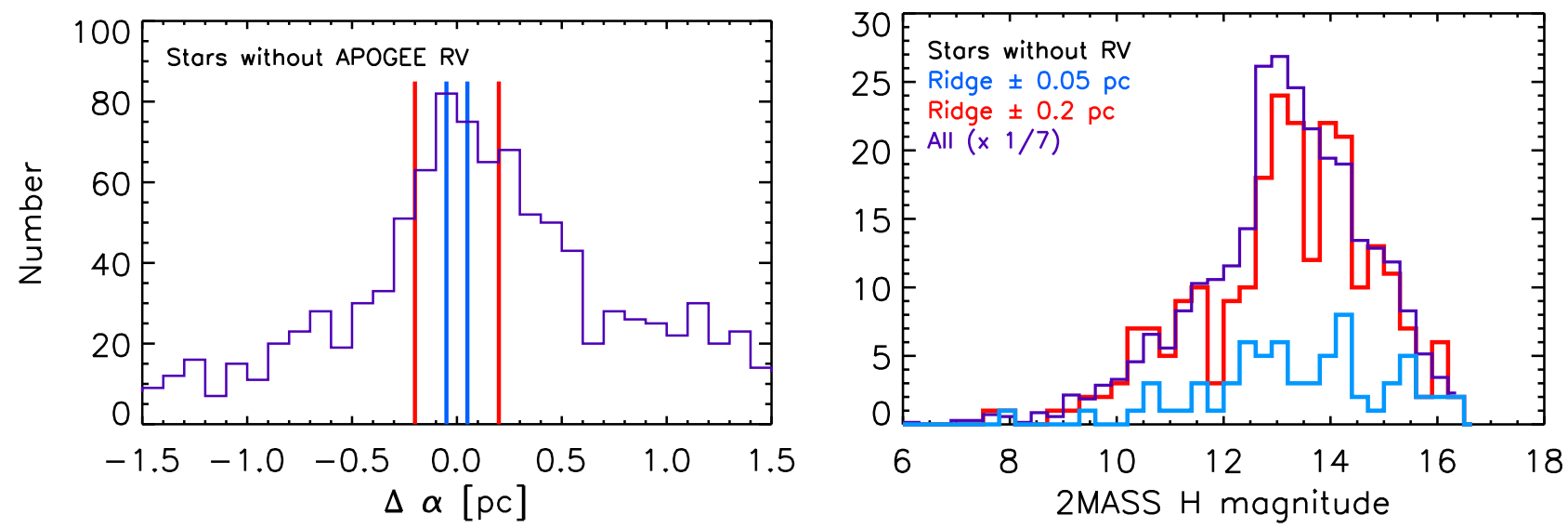

Fig. 12. Left: histogram of Megeath et al. (2012) IR-excess (Class II) stars that lack Apogee RVs as a function of distance from the filamentary spine. Subsets within 0.2 and $0.05 \mathrm{pc}$ are indicated. Right: $H$-band magnitude distribution of these three populations, with the whole population scaled down by a factor 7 for ease of comparison. Full population and $0.2 \mathrm{pc}$ subsample are statistically indistinguishable, nor is there any obvious bias from the $H<12.5$ Apogee selection in the 0.05 pc subsample.
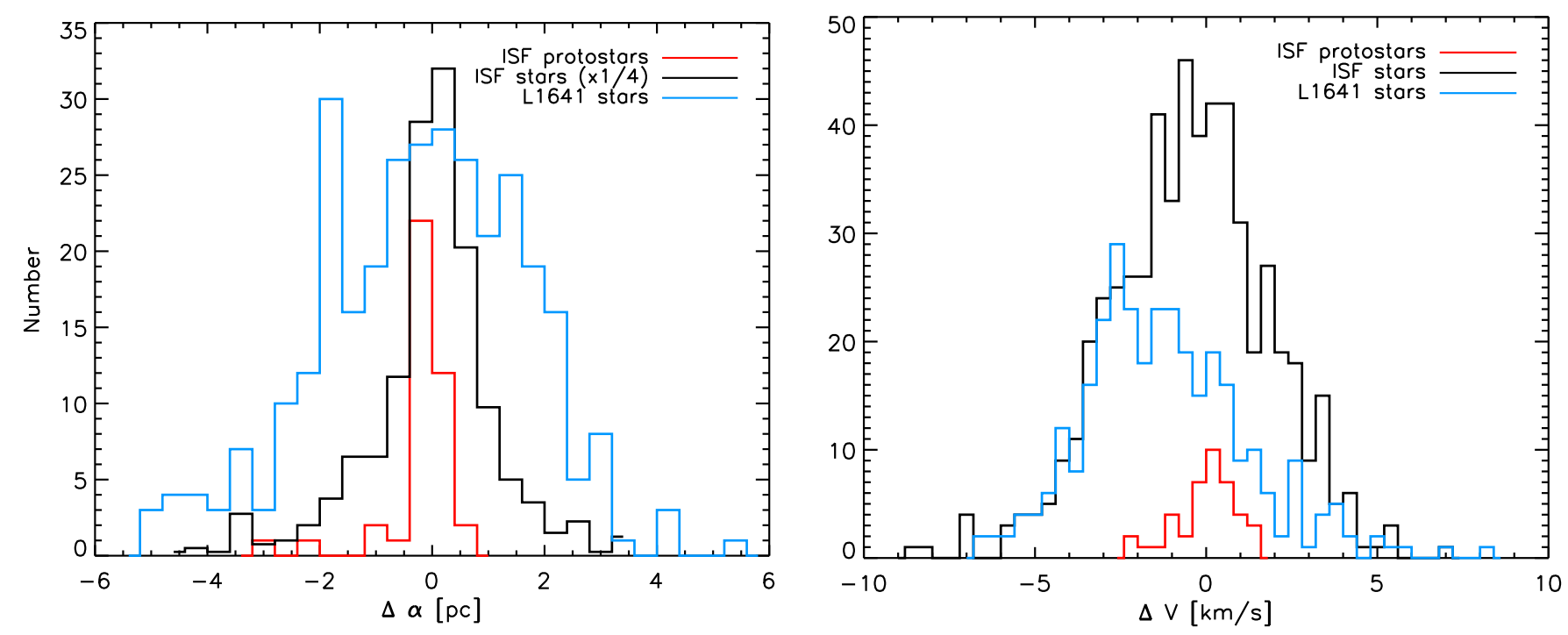

Fig. 13. Histograms of offsets from spatial (left) and velocity (right) ridgelines for three populations ISF protostars (red), ISF stars (cyan) and L1641 stars (black). For the two stellar populations, the ratios of the mean amplitudes of deviation given by Eq. (18) yield age estimates.

not the case, with lumpiness being most prominent in the protostar panel, but quite prominent in the L1641 panel as well, which in particular has a severe velocity asymmetry.

Another way to approach this issue is through the relations derived in Sect. 3. Recall that for well mixed orbits, $\langle r\rangle \simeq$ $r_{\max } / \sqrt{2}$ and $\langle v\rangle=1.2 \mathrm{~km} \mathrm{~s}^{-1}\left(r_{\max } / \mathrm{pc}\right)^{3 / 16}$. For quantities observed in projection (i.e., those shown in Fig. 10) we expect both quantities to be reduced by $\sqrt{2}$. Fig. 13 shows histograms of these offsets for all three populations. Numerically we find mean projected quantities

$\left\langle r_{\perp}\right\rangle=(0.38,0.81,1.48) \mathrm{pc} ; \quad\left\langle v_{\|}\right\rangle=(0.64,1.81,2.21) \mathrm{km} \mathrm{s}^{-1}$

for the ISF protostars, ISF stars, and L1641 stars, respectively. Here we have subscripted the two variables to emphasize that they refer to different Cartesian directions.

If, for example, the ISF stars were a well-mixed population, then typically $r_{\max } \sim 2 * 0.81 \mathrm{pc}=1.6 \mathrm{pc}$. Hence, it is possible to predict $\left\langle v_{\|}\right\rangle \sim 1.2 / \sqrt{2}(1.6)^{3 / 16}=0.9 \mathrm{~km} \mathrm{~s}^{-1}$, which is half the observed value. For the L1641 stars, the corresponding numbers are $r_{\max }=3.0 \mathrm{pc}$ and $\left\langle v_{\|}\right\rangle=1.05 \mathrm{pc}$, i.e., less than half the observed value. From this we can conclude that both groups of stars have typically not yet reached their first apogee after leaving the spine of the filament. We estimate mean ages (since ejection and consequent termination of protostellar Class I phase) of these populations as $0.81 \mathrm{pc} /\left(1.81 \mathrm{~km} \mathrm{~s}^{-1}\right)=0.44 \mathrm{Myr}$ and $1.48 \mathrm{pc} /\left(2.21 \mathrm{~km} \mathrm{~s}^{-1}\right)=0.65 \mathrm{Myr}$.

We note that these mean age estimates could be corrupted in various ways. For example, sources in binaries would have a random component added to their motion. This cannot be a dominant effect since it would obliterate the clumpiness of the phase-space diagrams if it were. However, it could artificially depress the age estimate. The argument also assumes that ejections from the filament are occurring at random angles relative to the filamentary axis, which might not be the case. In addition, the filament has accelerated by different amounts in different directions at each position along the filament since ejection. This adds noise (hence power) to both components. This effect should roughly cancel at first order but may still impact the estimates. 
The same method cannot be applied to the protostars partly because they lie too close to the filament in both position and velocity to allow precise measurements of their offsets and partly because the handful of protostars that form on sub-filaments are not being properly handled by the formalism. Indeed, the protostar panel shows intriguing evidence for extreme hugging of the filament. In particular, there is a strong knot of protostars within $\pm 0.3 \mathrm{~km} \mathrm{~s}^{-1}$ and $\pm 0.2 \mathrm{pc}$ of the origin. Since Class I protostars have estimated ages that are $\sim 3$ times loger than Class 0 protostars (e.g., Dunham et al. 2014) then we would expect to see substantial diferences between the two samples if we where observing a pure age effect. However, although the numbers are small, we find that the Class 0 and Class I protostars exhibit no clear diferences in projected quantities.

\section{Gravitational versus magnetic energy}

Normally the gravitational potential energy of a system is calculated by asking how much energy must be injected to separate its constituent particles to infinity. We will eventually have to make such an estimate, but of more immediate relevance to understanding the waves that are propagating in the filament is the energy required to lift all the material within some radius $r_{\max }$ that characterizes the filament oscillations to that radius. Recalling that $\rho(r)=(\gamma f / 2 \pi) K / \mathrm{pc}^{2}(r / \mathrm{pc})^{\gamma-2}$, and $\Lambda(r)=f K(r / \mathrm{pc})^{\gamma}$, we obtain

$$
\frac{\mathrm{d} E}{\mathrm{~d} l}=\int_{0}^{r_{\max }} \mathrm{d} r(2 \pi r \rho) 2 G \Lambda \ln \frac{r_{\max }}{r}=\frac{f^{2}}{\gamma} G K^{2}\left(\frac{r_{\max }}{\mathrm{pc}}\right)^{2 \gamma}
$$

We can now ask what is the rms magnetic field $B$ in this zone that is required to equal this potential energy per unit length, i.e., $\left\langle B^{2}\right\rangle \pi r_{\max }^{2} / 8 \pi$. We find

$$
\sqrt{\left\langle B^{2}\right\rangle}=\sqrt{\frac{8 G}{\gamma}} f \frac{K}{\mathrm{pc}}\left(\frac{r_{\mathrm{max}}}{\mathrm{pc}}\right)^{\gamma / 2-1} \rightarrow 70 \mu G\left(\frac{r_{\mathrm{max}}}{\mathrm{pc}}\right)^{-13 / 16}
$$

This value is consistent with measurements by Heiles (1997) at of order $1 \mathrm{pc}$ from the filament. Equality of magnetic field and gravitational potential energy would imply zero total energy on these scales, even without considering kinetic energy due to turbulence, and therefore would virtually guarantee instabilities. Such instabilities could plausibly trigger and sustain the transverse waves through the filament that are indicated by its morphology, its kinematics, and the quasi-periodic series of star-formation bursts in the north.

In general, one concern is that subcritical fields would blow the system apart, perhaps allowing one burst of star formation but not repeated ones. However, the magnetic fields are only subcritical on scales of the observed undulations. As we have shown in Sect. 2, the filament extends to much larger radii than its easily visible spine. Although we do not know the full extent, we can place a lower limit on the ratio of total gravitational binding energy to the local one by evaluating Eq. (19) at $8.5 \mathrm{pc}$ and $1 \mathrm{pc}$

$$
\frac{(\mathrm{d} E / \mathrm{d} l)_{8.5}}{(\mathrm{~d} E / \mathrm{d} l)_{1}}=8.5^{2 \gamma}=5.0
$$

where $l$ is length along the filament. Hence, it is quite possible for the magnetic fields to be subcritical on the scales of the filament oscillations and supercritical on scales of the filament as a whole.

\section{Discussion}

Starting from a new measurement (the first ever) of the gravitational potential of Orion A, and making use of information about the kinematics of the gas, protostars, and stars, as well as the magnetic fields, available from the literature, we have argued for a new mechanism of star formation in the integral shaped filament (ISF), namely, the slingshot mechanism.

The slingshot arises specifically because the magnetic fields in this region are subcritical on transverse scales of $\sim 1 \mathrm{pc}$, leading to instabilities of two types. First, on the (longitudinal) scale of the filament as a whole $(\sim 10 \mathrm{pc})$, there are global instabilities that result in a transverse wave that is apparent in the positional and RV data. Second, there are local pinching instabilities that generate repeated episodes of rapid star formation, one of which is ongoing in the ONC, with previous episodes having left behind the NGC 1977 and NGC 1981 clusters. Protostars form along the filament due to the high density of gas on its ridgeline and are initially accelerated transversely with the filament as it undergoes transverse oscillations. This continues so long as the protostars remain coupled to the gas. Eventually, the mass of the protostars becomes too great and they decouple. At this point, they continue to move with whatever transverse velocity the filament had at the time of decoupling, while the filament itself continues to oscillate.

The global instability (leading to the transverse wave) is long lived because the magnetic fields are supercritical on scales of the potential ( $>8 \mathrm{pc}$ transverse). Hence, it survives many episodes of star formation (as traced by the clusters we can seeNGC 1977 and 1981 - and probably older ones that have subsequently dispersed). The local instabilities are terminal: they result in the consumption and/or dispersal of the gas that is pinched off and are responsible for the gradually growing gap between Orion A and Orion B to the north.

Further south is L1641, which shares the same large-scale potential as the ISF (see Fig. 5), but has a significantly shallower profile at transverse separations $b<2.5 \mathrm{pc}$. In contrast to the ISF, it does not exhibit transverse-wave morphology and it is not forming stars at a rapid rate (Stutz \& Kainulainen 2015). In these respects, it is more like Taurus and other nearby, lower-mass, molecular clouds.

That is, in our view, L1641 represents a first stage of star formation, characterized by straight, magnetically supercritical filaments with low star formation rates, whereas the ISF represents a second stage, characterized by higher star formation rates and driven by magnetic instabilities that give rise to transverse waves. Thus, just as the ISF's future is foretold by NGC 1977 and 1981, so the future of L1641 is to collapse into a magnetically dominated filament with high star formation rate.

However, in contrast to L1641, not all first-stage filaments will reach the second stage. L1641 can do so only because of its deep potential well. In this picture, lower-mass clouds like Taurus will disperse without reaching the second stage.

Although this picture is completely new, it is largely consistent with previous work on molecular clouds, both theoretical and observational, as we now review.

\subsection{Two mass scales for molecular clouds}

To compare our measurements of the mass profiles of the ISF and L1641 with those of more nearby filaments that have been reported in the literature, we focus on the projected mass per unit length within $b<0.1$ pc. From Eq. (3), we find $\lambda(0.1 \mathrm{pc})=$ $160 M_{\odot} / \mathrm{pc}$. Because L1641 has a similar profile for $b>2.5 \mathrm{pc}$ 
but a shallower power law $(\gamma=1 / 2$ vs. $\gamma=3 / 8$ for $b<2.5 \mathrm{pc})$ we estimate that the L1641 line density is lower by a factor $(2.5 / 0.1)^{1 / 2-3 / 8}=1.5$. That is, $\lambda(0.1 \mathrm{pc})=107 M_{\odot} / \mathrm{pc}$.

For Taurus, we derive $\lambda(0.1 \mathrm{pc})=50 M_{\odot} / \mathrm{pc}$ from Fig. 5 of Palmeirim et al. (2013). For Musca, we derive $\lambda(0.1 \mathrm{pc})=$ $25 M_{\odot} /$ pc from Fig. 5 of Kainulainen et al. (2016). That is, the ISF has a factor 3-6 higher line density that these nearby filaments. It can be further contrasted in that it has a much higher star formation rate than Taurus (and, of course, than Musca, which is not forming stars at all). Most strikingly, Taurus and Musca are straight, whereas the ISF is integral shaped. Moreover, we detect no turnover in the profile at our resolution limit of $F W H M \sim 0.04 \mathrm{pc}$, whereas more nearby clouds, including Taurus and Musca, typically show FWHM $\sim 0.1 \mathrm{pc}$ (see also e.g., André et al. 2014; Arzoumanian et al. 2011; Könyves et al. 2015). Hence, this is another potential difference, although this issue must be more carefully explored. Finally, it appears that the density profiles of more nearby clouds tend to cluster near $r^{-2}$ (also see Alves et al. 1998; Lada et al. 1999) compared to $r^{-13 / 8}$ for the ISF. However, due to the somewhat irregular behavior of the nearby clouds as well as relatively large errors in their measurement, this comparison cannot yet be made rigorously.

Hence, with respect to all quantities and features that can be reliably measured, the ISF is distinct from other nearby clouds. This is consistent with (although it by no means proves) our suggestion that the ISF represents a different phase of star formation. In this picture, L1641 is intermediate, being governed by the same large-scale potential, but not having (yet) evolved to the second phase represented by the ISF.

To find analogs of the ISF, we must search to greater distances. For example, G11 has a line density of $\lambda=600 M_{\odot} / p c$ out to a column density of $\Sigma \geq 20 M_{\odot} / \mathrm{pc}^{2}$ (Kainulainen et al. 2013). To compare to the ISF, we consider an impact parameter of $b=7.7 \mathrm{pc}$, which matches the same $\Sigma$ limit. We then find $\lambda(7.7 \mathrm{pc})=830 M_{\odot} / \mathrm{pc}$ for the ISF. This is qualitatively similar to G11. Hence, while the ISF probably has many analogs, essentially all of them are far more difficult to study.

\subsection{NGC 1333 in Perseus: an ISF analog?}

Data and analysis by Foster et al. (2015) shows that NGC 1333 in Perseus has a number of features that are remarkably similar to the ISF. First, their Fig. 5 shows RV oscillations, such as would be expected from a transverse wave. Second, the dense cores (Kirk et al. 2007) in this figure tightly follow this gas profile (just as the protostars do in the ISF), while the stars show greater dispersion (just as the ISF stars). In addition, the line density in the filament may be comparable to the ISF. Like the ONC/ISF system, NGC 1333 is a dense cluster embedded in a linear filament running north-south (see Fig. 14). However, in contrast to the ISF, there is an important secondary east-west filament intersecting the primary filament. Perhaps for this reason, Foster et al. (2015) have analyzed the profile using radial averages, so a precise quantitative comparison of NGC 1333 and the ISF is not yet possible.

Foster et al. (2015) posit that the NGC 1333 dense cores may be coupled to the magnetic fields, but do not suggest (as we have for the ISF) that they inherit the magnetically accelerated gas motions when they are released. Instead, they argue that when the cores evolve into stars, they directly acquire velocities characteristic of the potential by some unspecified process.

Foster et al. (2015) have combined Herschel 160, 250, 350, $500 \mu \mathrm{m}$ data to create a dust-column map of Perseus, similar to the one made by Stutz et al. (2015) for Orion A, which was

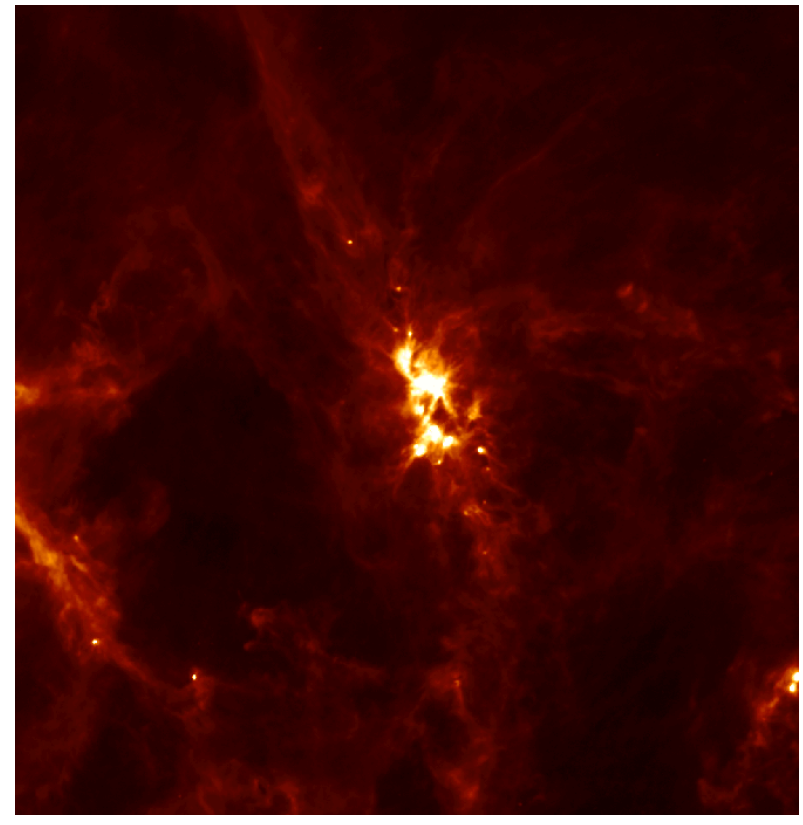

Fig. 14. Herschel $250 \mu \mathrm{m}$ image of the NGC 1333 region of Perseus, the scale is $6.1 \mathrm{pc}$ on a side.

used in this paper. It would be straightforward to reconstruct the potential using such a map. We note that CephOB3, with a gas morphology dominated by a main filament and clusters forming at each edge of the break in the filament, may be a promising ISF analog. See Fig. 2 of Gutermuth et al. (2011).

\subsection{Molecular cloud formation}

Low mass molecular clouds are characterized by straight, sometimes intersecting filaments. For example, Palmeirim et al. (2013) observe a large straight filament in Taurus (B211/3), with a radial density profile $\rho \propto r^{-2 \pm 0.4}$ between $r=0.05 \mathrm{pc}$ to $4 \mathrm{pc}$ and mass per unit length $\$ 45 M_{\odot} / \mathrm{pc}$. For example, the Aquila, Lupus, and IC 5146 filaments (Könyves et al. 2015; Benedettini et al. 2015; Arzoumanian et al. 2011) also appear fairly straight.

Such structures are readily produced in simulations of turbulent gas with and without magnetic fields (Padoan 1995; Mac Low \& Klessen 2004; Federrath 2016; Smith et al. 2014, 2016; Kirk et al. 2015; Chen \& Ostriker 2015). The fact that plausible representations of filaments can be produced even without magnetic fileds makes turbulence a leading candidate for the formation of molecular clouds.

Of course, the situation cannot be so simple because magnetic fields are observed to be almost universally associated with filaments. For example, Palmeirim et al. (2013) observed striations perpendicular to the Taurus B211/3 filament and argued that these were tracing mass accretion. These striations are approximately parallel to the projected plane of the sky magnetic field, as traced by optical polarization (Chapman et al. 2011; Heiles 2000; Heyer et al. 2008). Fiege \& Pudritz (2000a,b) argue that such a shallow $\left(r^{-2}\right)$ density profile as that estimated in B211/3 would be consistent with models of filaments in isothermal equilibrium that are threaded by helical magnetic fields.

However, the ubiquity of magnetic fields is not necessarily inconsistent with the magnetically-free turbulent simulations. It could be that when filaments form out of the ISM, they contain magnetic fields but these are subdominant. Hence, simulations 
need not take account of them to get the basic structure right. Then later, as gas accretion concentrates the field lines, the magnetic fields play an increasingly central role.

This would be consistent with our picture, in which magnetic fields play a qualitatively greater role in the second stage of star formation (illustrated by the ISF) than in the first stage, as exemplified by L1641 (e.g., Polychroni et al. 2013) and also the lower-mass, nearby clouds. These, indeed, all have nearly straight-line filaments. While they may have magneticallymediated flows, none show evidence for the violent magnetic instabilities that characterize the ISF.

And, in conformity with this, no turbulence simulation has ever produced a filament that appears integral shaped. Moreover, it is a definite prediction from our picture that none ever will, whether they include magnetic fields or not. We argue that the ISF is a specific product of internal evolution of the cloud, and cannot be directly produced by any combination of initial conditions, with or without magnetic fields.

\subsection{Molecular cloud lifetimes}

Hartmann et al. (2001) and Ballesteros-Paredes \& Hartmann (2007) argue that lifetimes of nearby low mass molecular clouds are $\sim 1$ to $3 \mathrm{Myr}$. These lifetime estimates hinge on two facts. First, the stars forming in these clouds have ages of order $\sim 1$ to $3 \mathrm{Myr}$ and not more (Jeffries et al. 2011). Second, of all clouds having qualitatively similar surface densities and masses, roughly two-thirds are forming stars. Hence, clouds must start forming stars soon after they coalesce and they must disperse soon after they start forming stars.

In seeming contrast, Murray (2011; see also Meidt et al. 2015) measures lifetimes $27 \pm 12 \mathrm{Myr}$ for massive molecular clouds. However, he argues that there is no real conflict. Partly he takes issue with stellar-based age estimates of the lower mass clouds, but his main point is that the cloud masses are an order of magnitude larger, while their densities are a factor of a few lower, leading to longer infall times and so lifetimes.

Within the framework of our picture, these results are consistent for a fundamentally distinct, though related, reason. The low-mass clouds that were the basis of the Hartmann et al. (2001) study have insufficient mass to form (or to survive the formation of) magnetically dominated filaments. Hence, they are dispersed as soon as they start forming stars. The massive clouds studied by Murray (2011) do form such structures. As a result, they are capable of the higher rates of star formation, such as that seen in the ISF, and this is the reason that they are noticed despite their typical distances of several kpc. However, because they are so far away, they cannot be studied in the same detail as Orion A, and hence the fact that they are in a second phase of star formation has never been recognized.

\subsection{Violent acceleration: magnetic fields vs. cold collapse}

We have shown that the ISF stars are moving substantially more rapidly and display much less clumping than ISF protostars. Foster et al. (2015) report similar results for NGC 1333 in Perseus. Somehow, stars are being violently accelerated post birth. The question is how?

To date, there are only two theories that purport to explain this. We have proposed a slingshot mechanism by which magnetically driven instabilities lead to ejection of protostars from an oscillating filament. Hartmann \& Burkert (2007) have proposed violent relaxation following cold collapse of a starforming cloud of gas (see also Tobin et al. 2009).

How do these theories relate to the facts? First, all of the available evidence is consistent with filaments being wrapped in toroidal magnetic fields. In all star-forming filaments that have been studied, the transverse fields (traced by polarization) are roughly perpendicular to the filament, which is exactly what is expected from toroidal fields. For the ISF, the line of sight fields change direction as one crosses the filament, which is also the expected behavior. The energy of the magnetic field is comparable to the gravitational potential and so is high enough to generate instabilities. The filament's appearance is consistent with it undergoing a transverse wave. Such an accelerating filament will naturally hold on to diffuse material that is in the process of forming a protostar and will equally naturally release it once the protostar becomes sufficiently compact.

In cold collapse, the protostars must also form on filaments. Otherwise we live in a special epoch, not only for the ISF but also NGC 1333. These filaments then must have subsequently disappeared, being replaced by the filament that we can currently see, which is one of the most prolific in the nearby universe. In addition, simulations carried out by Kuznetsova et al. (2015) show that the sink particles (representing protostars) do not cluster in phase space like the real ISF protostars (see their Figs. 1, 2, and 6 and our Fig. 6).

It is possible to counter that if there were strong toroidal fields, then these would have prevented formation of the filament in the first place by blocking infall. Our answer: subcritical magnetic fields are there. Heiles (1997) measured their amplitude $20 \mathrm{yr}$ ago and we have now shown they are subcritical by measuring the potential. Such high field strengths are naturally explained by magnetic compression of gas due to currents. That is, the material gets to its current position not by crossing field lines but by compressing them. It may be objected that this process would lead to pinching instabilities. Our answer: yes, pinching instabilities are expected and this is exactly what leads to cluster formation.

\section{Conclusions}

Systematic review of publicly available data on Orion A has led to striking conclusions. Some of these follow directly from the observations. The surface density of the filament declines as a power law as one moves away from its spine, which itself displays previously well-known undulations. The regularity of the larger structure already tells us that the dense spine at its center is being subjected to strong transverse perturbing forces, since its relaxation time is much shorter than that of the larger, regular structure. The undulations are present in both the position and radial velocity of the spine.

These undulations appear to be ejecting protostars, which is probably the mechanism for shutting off their accretion. The evidence for this is that protostars, virtually without exception, all lie projected directly on filaments and their radial velocities are consistent with those of this filamentary gas at $<1 \mathrm{~km} \mathrm{~s}^{-1}$, while for stars the opposite is the case: they are generally both off filaments and have radial velocities that differ from the neighboring filaments by several $\mathrm{km} \mathrm{s}^{-1}$. Of course, this argument can be directly applied only to the subset with RV measurements, and this subset could in principle have been biased against stars inside filaments due to higher extinction. However, our investigation showed that any such bias must be very weak.

The relatively high ratio of velocity to physical offsets of ISF stars from their respective ridgelines leads to a relatively low 
age estimate $\sim 0.44 \mathrm{Myr}$, where age means time since ejection from the filament and consequent termination of the Class I protostar phase. This is consistent with other evidence of their youth such as the high ratio of protostars to stars and the functional form of the column-density histogram (Stutz \& Kainulainen 2015).

The youth of the ISF is of particular interest because it fits into a larger pattern. The ISF sits at the northern end of the Orion A filament, where end means end of a discernible spine of gas. Yet just $1.6 \mathrm{pc}$ from the northern tip of the ISF (4.1 pc from the ISF center) is the young cluster NGC 1977, which we dub the orphan cluster because the gas column from which it formed is no longer present. The cluster is predominantly composed of disk sources (Class II stars), from which we estimate an age of 2 Myr. Moreover, 2.9 pc further north is an older cluster, which Pillitteri et al. (2013) showed is predominantly composed of photospheric (Class III) stars, with a minority of Class II stars. Hence, it is yet older, perhaps 4 Myr. Thus we see the presence and/or vestiges of three roughly periodic episodes of star formation, each of which took place at the northern end of the Orion A filament as it existed at the epoch of that cluster's formation. Although there is no direct evidence for extrapolating this trend, it is possible to guess that these quasi-periodic episodes of cluster formation stretch back further in time and are responsible for the progressive dispersal (and consumption) of the gas filament that used to extend all the way to Orion B. Indeed, we see some evidence for dispersal of the gas associated with the orphan cluster in the westward trail of gas that we have dubbed the ghost filament.

In order to assess the cause of these periodic bursts of cluster formation, we construct the simplest possible model: we assume that the observed surface density power law $\Sigma(b) \propto b^{-5 / 8}$ is due to an underlying axisymmetric density profile $\rho(r) \propto r^{-13 / 8}$. It is then straightforward to determine the integrated line density as a function of cylinder radius, the potential energy, etc. We find that the line density of gravitational potential energy on $1 \mathrm{pc}$ scales is comparable to the line density of magnetic energy as measured by Heiles (1997) on similar scales. That is, the magnetic fields are subcritical, which naturally leads to repeated perturbations propagating through the filament as mainly traverse waves. We also show that while the magnetic fields are subcritical on the $1 \mathrm{pc}$ scales of the observed undulations of the filament, they are supercritical on the scales of the extended regular structure, which we trace to at least 8.5 pc. Thus, the spine can be subjected to violent periodic perturbations, while at the same time surviving many such episodes.

It is the transverse acceleration of the filament spine that is responsible for "ejecting" the protostars via a slingshot mechanism. That is, it is not the protostars that leave the gas but the gas that leaves the protostars. As long as the protostars are entrained in extended envelopes that are mechanically linked to the larger spinal column, the cores at their centers are dragged along with the column as it accelerates. However, when the cores reach sufficient mass relative to the larger envelope, their own inertia prevents them from following the gas as it accelerates. See Fig. 2 for a cartoon illustration of this process. If this phase occurs at a time of low acceleration, then even a tenuous connection between the core and the envelope is sufficient to keep the protostar in the column. However, at times of high acceleration, ejection can occur at an earlier phase of protostar evolution.

We therefore predict that the initial launching velocities of the protostars should be of order the semi-amplitude of the observed undulations of the spine, namely $\sim 2.5 \mathrm{~km} \mathrm{~s}^{-1}$. Given the potential $\Phi(r)=6.3\left(\mathrm{~km} \mathrm{~s}^{-1}\right)^{2}(r / \mathrm{pc})^{3 / 8}$, this implies maximum excursions of $r_{\max } \sim\left(2.5^{2} / 2 \times 6.3\right)^{8 / 3} \sim 4.0 \mathrm{pc}$, which are achieved after a time $\Delta t \sim 4.8 \mathrm{Myr}$. Thus, most recently formed stars should be on their first ascent away from their birth filament, a prediction that could be confirmed by Gaia proper motions.

The timescales of these filament motions can be estimated from the ratio of spatial to velocity amplitudes, namely $\sim 1.5 \mathrm{pc} /\left(2.5 \mathrm{~km} \mathrm{~s}^{-1}\right) \sim 0.6 \mathrm{Myr}$. Hence, the accelerations are $a \sim$ $4 \mathrm{~km} \mathrm{~s}^{-1} \mathrm{Myr}^{-1}$. This is similar to the acceleration at $\sim 0.05 \mathrm{pc}$ from a $\sim 2 M_{\odot}$ protostar. Since the filament ridges have similar widths to the first number and protostar envelopes have similar masses to the second, it may be that it is the amplitude of these undulations that sets the stellar mass scale.

Acknowledgements. We thank James Di Francesco for providing the $\mathrm{NH}_{3}$ data in advance of publication. We thank Boaz Katz, Ralf Pudritz, and Martin Pessah for excellent discussions that improved this work. We thank the anonymous referee for a helpful report. We thank Melissa Ness, Sarah Sadavoy, Tom Megeath, Lee Hartmann, and Andrey Kravtsov for informative and helpful discussions. A.G. is grateful for the hospitality of the MPIA and acknowledges support from NSF AST 1516842. This paper includes data from Herschel, a European Space Agency (ESA) space observatory with science instruments provided by European-led consortia and with important participation from NASA. We acknowledge the use Digitized Sky Survey data in this work. The full DSS acknowledgement can be found at https://archive.stsci.edu/dss/ acknowledging.html We acknowledge the use of SDSSIII data in this work. The full SDSSIII acknowledgement can be found at https://www.sdss3.org We acknowledge the use of the Georgia Institute of Technology website Edward Emerson Barnard's Photographic Atlas of Selected Regions of the Milky Way. The full acknowledgement can be found at http://www.library.gatech. edu/barnard/credits.html

\section{References}

Alves, J., Lada, C. J., Lada, E. A., Kenyon, S. J., \& Phelps, R. 1998, ApJ, 506, 292

André, P., Men'shchikov, A., Bontemps, S., et al. 2010, A\&A, 518, L102

André, P., Di Francesco, J., Ward-Thompson, D., et al. 2014, Protostars and Planets VI, 27

Arzoumanian, D., André, P., Didelon, P., et al. 2011, A\&A, 529, L6

Ballesteros-Paredes, J., \& Hartmann, L. 2007, Rev. Mex. Astron. Astrofis., 43, 123

Bally, J., Langer, W. D., Stark, A. A., \& Wilson, R. W. 1987, ApJ, 312, L45

Barnard, E. 1905, PLATE 41, In Aguila, Northwest of Altair

Benedettini, M., Schisano, E., Pezzuto, S., et al. 2015, MNRAS, 453, 2036

Chapman, N. L., Goldsmith, P. F., Pineda, J. L., et al. 2011, ApJ, 741, 21

Chen, C.-Y., \& Ostriker, E. C. 2015, ApJ, 810, 126

Da Rio, N., Tan, J. C., Covey, K. R., et al. 2016, ApJ, 818, 59

Dunham, M. M., Stutz, A. M., Allen, L. E., et al. 2014, Protostars and Planets VI, 195

Federrath, C. 2016, MNRAS, 457, 375

Fiege, J. D., \& Pudritz, R. E. 2000a, MNRAS, 311, 85

Fiege, J. D., \& Pudritz, R. E. 2000b, MNRAS, 311, 105

Foster, J. B., Cottaar, M., Covey, K. R., et al. 2015, ApJ, 799, 136

Furlan, E., Megeath, S. T., Osorio, M., et al. 2014, ApJ, 786, 26

Furlan, E., Fischer, W. J., Ali, B., et al. 2016, ApJS, accepted

[arXiv: 1602.07314]

Gutermuth, R. A., Pipher, J. L., Megeath, S. T., et al. 2011, ApJ, 739, 84

Hacar, A., Alves, J., Forbrich, J., et al. 2016, A\&A, 589, A80

Hartmann, L., \& Burkert, A. 2007, ApJ, 654, 988

Hartmann, L., Ballesteros-Paredes, J., \& Bergin, E. A. 2001, ApJ, 562, 852

Heiles, C. 1997, ApJS, 111, 245

Heiles, C. 2000, AJ, 119, 923

Heyer, M., Gong, H., Ostriker, E., \& Brunt, C. 2008, ApJ, 680, 420

Jeffries, R. D., Littlefair, S. P., Naylor, T., \& Mayne, N. J. 2011, MNRAS, 418, 1948

Johnstone, D., \& Bally, J. 1999, ApJ, 510, L49

Kainulainen, J., Ragan, S. E., Henning, T., \& Stutz, A. 2013, A\&A, 557, A120

Kainulainen, J., Hacar, A., Alves, J., et al. 2016, A\&A, 586, A27

Kant, E. 1755, Universal Natural History and Theory of Heaven (2009 Richer Resources Publications)

Kirk, H., Johnstone, D., \& Tafalla, M. 2007, ApJ, 668, 1042

Kirk, H., Klassen, M., Pudritz, R., \& Pillsworth, S. 2015, ApJ, 802, 75

Könyves, V., André, P., Men'shchikov, A., et al. 2015, A\&A, 584, A91 
Kuznetsova, A., Hartmann, L., \& Ballesteros-Paredes, J. 2015, ApJ, 815, 27

Lada, C. J., Alves, J., \& Lada, E. A. 1999, ApJ, 512, 250

Lang, D. 2014, AJ, 147, 108

Lombardi, M., Bouy, H., Alves, J., \& Lada, C. J. 2014, A\&A, 566, A45

Mac Low, M.-M., \& Klessen, R. S. 2004, Rev. Mod. Phys., 76, 125

Majewski, S. R., Schiavon, R. P., Frinchaboy, P. M., et al. 2015, AJ, submitted [arXiv: 1509.05420]

Matthews, B. C., \& Wilson, C. D. 2000, ApJ, 531, 868

Megeath, S. T., Gutermuth, R., Muzerolle, J., et al. 2012, AJ, 144, 192

Meidt, S. E., Hughes, A., Dobbs, C. L., et al. 2015, ApJ, 806, 72

Murray, N. 2011, ApJ, 729, 133

Ness, M., Hogg, D. W., Rix, H.-W., Ho, A. Y. Q., \& Zasowski, G. 2015, ApJ, 808,16

Nishimura, A., Tokuda, K., Kimura, K., et al. 2015, ApJS, 216, 18

Padoan, P. 1995, MNRAS, 277, 377

Palmeirim, P., André, P., Kirk, J., et al. 2013, A\&A, 550, A38

Peterson, D. E., \& Megeath, S. T. 2008, Handbook of Star Forming Regions, Vol. I, 590

Pezzuto, S., Elia, D., Schisano, E., et al. 2012, A\&A, 547, A54
Pillai, T., Kauffmann, J., Tan, J. C., et al. 2015, ApJ, 799, 74 Pillitteri, I., Wolk, S. J., Megeath, S. T., et al. 2013, ApJ, 768, 99 Poidevin, F., Bastien, P., \& Matthews, B. C. 2010, ApJ, 716, 893 Poidevin, F., Bastien, P., \& Jones, T. J. 2011, ApJ, 741, 112 Polychroni, D., Schisano, E., Elia, D., et al. 2013, ApJ, 777, L33 Ragan, S., Henning, T., Krause, O., et al. 2012, A\&A, 547, A49 Ripple, F., Heyer, M. H., Gutermuth, R., Snell, R. L., \& Brunt, C. M. 2013, MNRAS, 431, 1296

Safron, E. J., Fischer, W. J., Megeath, S. T., et al. 2015, ApJ, 800, L5

Smith, R. J., Glover, S. C. O., \& Klessen, R. S. 2014, MNRAS, 445, 2900

Smith, R. J., Glover, S. C. O., Klessen, R. S., \& Fuller, G. A. 2016, MNRAS, 455,3640

Stutz, A. M., \& Kainulainen, J. 2015, A\&A, 577, L6

Stutz, A., Launhardt, R., Linz, H., et al. 2010, A\&A, 518, L87

Stutz, A. M., Tobin, J. J., Stanke, T., et al. 2013, ApJ, 767, 36

Tatematsu, K., Kandori, R., Umemoto, T., \& Sekimoto, Y. 2008, PASJ, 60, 407

Tatematsu, K., Ohashi, S., Sanhueza, P., et al. 2016, PASJ, 68, 24

Tobin, J. J., Hartmann, L., Furesz, G., Mateo, M., \& Megeath, S. T. 2009, ApJ, 697, 1103 